\title{
Erratum: A hybrid strong/weak coupling approach to jet quenching
}

\author{
Jorge Casalderrey-Solana, ${ }^{a}$ Doga Can Gulhan, ${ }^{b}$ José Guilherme Milhano, ${ }^{c, d}$ \\ Daniel Pablos $^{a}$ and Krishna Rajagopal ${ }^{b, e}$ \\ ${ }^{a}$ Departament d'Estructura $i$ Constituents de la Matèria and \\ Institut de Ciències del Cosmos (ICCUB), Universitat de Barcelona, \\ Martí i Franquès 1, 08028 Barcelona, Spain \\ ${ }^{b}$ Laboratory for Nuclear Science and Department of Physics, \\ Massachusetts Institute of Technology (MIT), \\ Cambridge, MA 02139, U.S.A. \\ ${ }^{c}$ CENTRA, Instituto Superior Técnico, Universidade de Lisboa, \\ Av. Rovisco Pais, P-1049-001 Lisboa, Portugal \\ ${ }^{d}$ Physics Department, Theory Unit, CERN, \\ CH-1211 Genève 23, Switzerland \\ ${ }^{e}$ Center for Theoretical Physics, Massachusetts Institute of Technology (MIT), \\ Cambridge, MA 02139, U.S.A. \\ E-mail: jorge.casalderrey@ub.edu, dgulhan@mit.edu, \\ guilherme.milhano@tecnico.ulisboa.pt, dpablos@ecm.ub.es, \\ krishna@mit.edu
}

ERrATUM TO: JHEP10(2014)019

ARXIV EPRINT: 1405.3864

After the publication of this work we have found a small mistake in our implementation of the Glauber Monte-Carlo for the collision geometry, which resulted in an incorrect distribution of the points in the transverse plane at which our jets were created, for collisions within each centrality bin. After correcting this, the fitted values of $\kappa_{\mathrm{sc}}, \kappa_{\text {rad }}$ and $\kappa_{\text {coll }}$ in the current version of this paper are slightly smaller than previously reported. This correction also results in small (hardly visible) changes to all the plots in this paper, which we have corrected. Neither the changes to the fitted values of the $\kappa$ 's nor the changes to the figures affect any discussion or any conclusions of this work; we have made no changes to the wording in any discussion of any figure or result. We have also reduced the $p_{\mathrm{T}}$-cut of our PYTHIA simulations from $70 \mathrm{GeV}$ to $50 \mathrm{GeV}$ to further reduce the sensitivity of our computations to this cut. 
ABSTRACT: We propose and explore a new hybrid approach to jet quenching in a strongly coupled medium. The basis of this phenomenological approach is to treat physics processes at different energy scales differently. The high- $Q^{2}$ processes associated with the QCD evolution of the jet from its production as a single hard parton through its fragmentation, up to but not including hadronization, are treated perturbatively following DGLAP evolution, to which we ascribe a spacetime structure. The interactions between the partons in the shower and the deconfined matter within which they find themselves lead to energy loss. The momentum scales associated with the medium itself (of the order of the temperature) and with typical interactions between partons in the shower and the medium are sufficiently soft that strongly coupled physics plays an important role in energy loss. We model these interactions using qualitative insights inferred from holographic calculations of the energy loss of energetic light quarks and gluons in a strongly coupled plasma, obtained via gauge/gravity duality. We embed this hybrid model into a hydrodynamic description of the spacetime evolution of the hot QCD matter produced in heavy ion collisions and confront its predictions with experimental results for a number of observables that have been measured in high energy jet data from heavy ion collisions at the LHC, including jet $R_{\mathrm{AA}}$ as a function of transverse momentum, the dijet asymmetry, and the jet fragmentation function ratio, all as functions of collision centrality. The holographic expression for the energy loss of a light quark or gluon that we incorporate in our hybrid model is parametrized by a stopping distance. We find very good agreement with all the data as long as we choose a stopping distance that is comparable to but somewhat longer than that in $\mathcal{N}=4$ supersymmetric Yang-Mills theory. For comparison, we also construct analogous alternative models in which we assume that energy loss occurs as it would if the plasma were weakly coupled. We close with suggestions of observables that could provide more incisive evidence for, or against, the importance of trongly coupled physics in jet quenching. 


\section{Contents}

1 Introduction 1

2 A hybrid approach to jet quenching 4

3 In-medium energy loss of energetic particles $\quad 8$

3.1 Parton energy loss at strong coupling from falling semiclassical strings 8

$\begin{array}{ll}3.2 & \text { Comparison with other approaches } \\ & 12\end{array}$

3.3 Perturbative benchmarks: radiative and collisional energy loss in a weakly coupled plasma

4 Monte Carlo implementation

5 Comparison with jet data $\quad 18$

$\begin{array}{lll}5.1 \text { Jet reconstruction and jet } R_{\mathrm{AA}} & 19\end{array}$

5.2 Dijet asymmetry 24

5.3 Jet $R_{\mathrm{AA}}$ and mean dijet asymmetry 26

5.4 Energy lost by individual partons within a jet 27

$\begin{array}{ll}5.5 & \text { Fragmentation function ratio } \\ & 28\end{array}$

6 Conclusions, discussion and a look ahead 31

6.1 Conclusions 31

6.2 Significance of the extracted parameters 32

6.3 Opportunities for improvements to our implementation 34

6.4 Distinctive species dependence and discriminating observables 37

\section{Introduction}

One of the most striking results obtained from heavy ion collisions at the Large Hadron Collider (LHC) is the strong suppression of high energy jets observed in $\mathrm{Pb}-\mathrm{Pb}$ collisions with a center of mass energy of $2.76 \mathrm{TeV}$ per nucleon-nucleon collision [1,2]. This suppression, commonly referred to as jet quenching, is due to the energy loss suffered by the components of the jets on their way out of the hot QCD medium formed in a high energy heavy ion collision. The phenomenon of jet quenching was discovered prior to the LHC measurements, without reconstructing individual jets, primarily via the strong reduction in the number of intermediate- $p_{T}$ hadrons in heavy ion collisions at RHIC relative to protonproton collisions $[3,4]$. Jet quenching has come to be seen as one of the most powerful experimentally accessible tools with which to analyze the properties of deconfined QCD matter. The large magnitude of the effects of energy loss observed in heavy ion collisions at 
the LHC, together with the ability to study the effects of energy loss on many properties of individually reconstructed jets, increases the potential of these probes to provide accurate medium diagnostics, provided the mechanism by which they interact with the medium can be understood with sufficient precision.

One of the reasons why high energy jets are superior to other probes is that their production occurs at very high energy scales, $Q \gg \Lambda_{Q C D}$, which guarantees that their production spectrum is under good theoretical control, since it can be determined via perturbative QCD. Similarly, many of the properties of jets in vacuum are also controlled by physics at high energy scales and are therefore well understood theoretically. Therefore, observed deviations of those properties in a heavy ion environment must be due to the interaction of the different jet components with the hot hadronic medium that the nascent jet traverses on its way out of the collision zone. In general, the interaction with the medium constituents will lead to the degradation of the jet energy, but the precise mechanism or mechanisms by which this occurs depend on the nature of the medium.

Although the production of a hard parton that will become a jet, and the fragmentation of that parton as it propagates, are controlled by weakly coupled physics at high momentum scales, the physics of the medium produced in experimentally realizable heavy ion collisions is not weakly coupled. At sufficiently high temperatures the quark-gluon plasma must be a weakly coupled plasma of quark and gluon quasiparticles. However, in the temperature range explored by current colliders, namely $T \sim 150-600 \mathrm{MeV}$, we know from the comparison of more and more precisely measured experimental observables to more and more sophisticated calculations of relativistic viscous hydrodynamics that the quark-gluon plasma produced in heavy ion collisions is a droplet of strongly coupled liquid that expands and flows collectively, hydrodynamically (For a review, see ref. [5]). This fact makes the quark gluon plasma a very interesting form of matter that has attracted the interest of scientists in other fields in which other forms of strongly coupled matter arise. However, this fact also complicates the theoretical understanding of the properties and dynamics of the medium rather significantly. For this reason, in recent years there has been a growing interest in strongly coupled techniques that can shed light on the dynamics of the liquid plasmas that arise as the hot deconfined phases of other non-Abelian gauge theories which have holographically dual descriptions as gravitational theories in $4+1$ dimensional spacetimes containing a black hole horizon. The simplest example to which this gauge/gravity duality has been applied is the plasma that arises at nonzero temperature in strongly coupled $\mathcal{N}=4$ supersymmetric Yang Mills (SYM) theory in the limit of a large number of colors $N_{c}$. Holographic analyses performed in this and other gauge theories have led to many qualitative insights into the properties of the QCD plasma, its dynamics in heavy ion collisions, and the dynamics of probe particles propagating through the strongly coupled plasma. (See ref. [6] for a review).

The way in which a high energy excitation interacts with a deconfined non-Abelian plasma is well understood in two extreme, and unrealizable, limits. At weak coupling, by which we mean at unrealizably high temperatures at which the coupling constant at the medium scale is small, perturbative analyses show reliably that the dominant mechanism of in-medium energy loss is the radiative process of stimulated gluon emission caused by 
the scattering of the high energy parton off particles in the medium [7-12]. The rate of emission of these radiated gluons forms the basis of most current analysis of jet modification in the environment produced in heavy ion collisions. (See refs. [13-16] for reviews.) In addition, many of these studies also include a second energy loss process, that is in principle subleading for very high energy partons, namely the elastic transfer of energy to medium constituents, referred to as collisional energy loss [17]. The second unrealizable limit is the limit in which the coupling constant is assumed to be large at all relevant energy scales. In this case, gauge/gravity duality has made it possible to use holographic calculations to analyze the way in which varied energetic probes have their energy degraded, and are otherwise modified, as they propagate through strongly coupled plasma [18-27]. (For a review, see ref. [6].) These computations provide detailed dynamical information on the energy loss processes in this limit. The intuition that comes from these calculations is phrased in terms of the dual gravitational description, rather than in terms of gauge theory degrees of freedom. While these two extremes each provide invaluable guidance to understanding energy loss processes in a heavy ion environment, because the medium is strongly coupled while much of the physics of jets is governed by weakly coupled high momentum physics, at least as they are currently constituted neither approach can capture all important aspects of the dynamics.

The main difficulty in understanding jet dynamics in a strongly coupled QCD medium resides in the interplay between physics at very different energy scales. After their production via a (very) hard scattering, jets relax their large initial virtuality down toward the hadronic scale via an evolution process of branching into a shower of partons. In vacuum, this fragmentation process is governed by the Dokshitzer-Gribov-Lipatov-Altarelli-Parisi (DGLAP) equation. This perturbative process is crucial to understanding most jet properties. In the medium, this evolution occurs while at the same time partons in the developing shower suffer many soft exchanges of momenta of order the medium temperature $T$, which alter the fragmentation pattern. Since the momenta transferred in these interactions are not large, this physics is not weakly coupled just as the physics of the medium itself is not weakly coupled. This means that a part of the dynamics of jets propagating through the medium produced in a heavy ion collision is out of the regime of validity of perturbative QCD. Thus, jets are multi-scale probes sensitive to both strongly and weakly coupled physics. In the long run, their description in controlled calculations will require either a strongly coupled approach to far-from-equilibrium dynamical processes in QCD or calculations done via gauge/string duality that incorporate asymptotic freedom at short distance scales or both. As, at present, neither seems on the horizon we must limit our goals. A successful phenomenological model that describes the modifications of jets in the medium, today, must be a hybrid model in which one can simultaneously treat the weakly coupled physics of jet production and hard jet evolution and the strongly coupled dynamics of the medium and the soft exchanges between the jet and the medium. In this work, we will put forward a phenomenological approach which combines different physics mechanisms at different scales. While there have been other attempts to combine results obtained from weak and strong coupling [28-31], our approach is distinct since it focusses on using different calculational frameworks at the different energy scales. 
This paper is organized as follows: we describe how we set up our hybrid approach in section 2. The interaction of partons with a strongly coupled medium is reviewed in section 3. In section 4 we discuss how to implement these ideas in a simple Monte Carlo simulation of jets in heavy ion collisions, using a hydrodynamic description of the spacetime dynamics of the medium. We use this implementation of our hybrid approach to determine several jet observables, which we confront with data on jet $R_{\mathrm{AA}}$, the dijet asymmetry and jet fragmentation function ratios in section 5 . In section 6 we reflect upon the successes and limitations of our hybrid approach and, in addition, suggest further observables that, if measured, could provide more incisive evidence for or against the importance of strongly coupled physics in jet quenching.

\section{$2 \quad$ A hybrid approach to jet quenching}

As we have stressed in the preceding Introduction, no single theoretical framework is currently available within which controlled calculations of all important aspects of jet quenching in heavy ion collisions can reliably be carried out. This is so since we must simultaneously describe the perturbative dynamics at short distances and the strongly coupled physics at the medium scale. We will therefore resort to phenomenological modeling of the main physical processes occurring during the propagation of high energy partons through strongly coupled plasma. To simplify our analysis, we will focus on high energy, high virtuality jets, since a large separation between the hard and medium scales allows us to better separate the treatment of these two regimes. In this section, we will spell out and motivate the main assumptions behind our model.

Our first assumption is that the exchange of momentum with the medium, which in the absence of coherence effects among several plasma constituents is of the order of the temperature $T$, is smaller than the virtuality of any of the jet partons at any stage of the evolution. For sufficiently high energy jets, this assumption is certainly valid at the early stages of the evolution process, but it becomes more questionable at the late stages, when the evolution approaches the hadronization scale. Fortunately, these late stages also happen at later times, when almost all the partons in the shower are outside of the medium [32]. Since these small momentum exchanges cannot lead to a significant variation of a parton's virtuality, we will assume that the splitting kernel at each point in the evolution is as in the vacuum. This motivates our second assumption: because each splitting that occurs as the original parton fragments happens at smaller distance scales than the medium can resolve, we assume that the splitting probabilities are as in vacuum. Keeping the splitting kernel unmodified implies, in particular, that, in a probabilistic approach, the emission probability at each step in the Markovian chain remains independent of the medium dynamics.

It will be important to return to the second assumption above in future work for at least two reasons. First, we will be assuming that the splitting probability is unmodified even as the partons lose some of their energy and will thus be neglecting the fact that even in vacuum the splitting kernel depends on parton energy (through Sudakov logs, which is to say via the phase space for splitting). Second, we will be neglecting the possibility of additional splitting induced by multiple soft exchanges with the strongly coupled plasma, 
which accumulate into a hard momentum transfer. As such an effect is known to be important in a weakly coupled plasma with point-like constituents, it will, in the future, be interesting to investigate how to incorporate it within the hybrid model we are setting up in the present paper. However, assuming the physics at the medium scale to be strongly coupled, as we shall do throughout, renders any such weakly coupled large momentum transfer processes, and their modification, subleading in their consequences.

We now wish to apply a prescription for how much energy each parton in the shower loses as it propagates through the medium. That means that we need to know the temperature of the medium in which a particular parton in the shower finds itself, which in turn means that we need to know where each parton in the shower is in space and time. The DGLAP evolution equations that describe the fragmentation of the parent parton and the evolution of the resulting shower are derived in perturbative QCD in momentum space. They contain little information about how the process of showering, and the attendant relaxation in the virtuality of the individual partons in the shower, develops in space and time. This space-time information is unimportant in vacuum physics, since the partonic components of the jet do not interact with anything; all they do is fragment and in describing the jet in the final state it is completely unimportant where and when each splitting happened. However, in a heavy ion environment before the shower emerges from the medium every parton in the shower interacts with the medium, and the medium itself changes as a function of space and time. We therefore need to know where and when each splitting occurs. Based on the analysis of soft gluon emission, most jet Monte Carlo studies assign a time to each rung of the evolution equation related to the formation time of the emitted gluon $\tau_{f}=2 \omega / k_{\perp}^{2}$. However, the detailed implementation varies from one Monte Carlo implementation to another, which gives a sense of the theoretical uncertainty concerning the space-time evolution that is common to all in-medium event generators. In this work, we will use the prescription of ref. [32] and assign a life-time to each rung of the decay chain (i.e. to each parton in the shower) determined from their virtuality $Q$ and energy $E$ as

$$
\tau=2 \frac{E}{Q^{2}},
$$

with the factor of two chosen such that in the soft limit it coincides with the standard expression for the formation time. We will also assume that the strong virtuality ordering in the QCD shower translates into time ordering, with the hardest splittings occurring first. This implies that the later stages of the evolution, for which the virtuality is closer to the hadronization scale, occur at later times.

In between any of the virtuality relaxing splittings, the partons in the jet propagate through the strongly coupled plasma. The momenta exchanged between these partons and the medium is of order the medium temperature, and therefore, for plasma temperatures not far above the deconfining transition, these momentum exchanges are not weakly coupled processes. This is where strongly coupled dynamics plays a role. From the point of view of the jet shower, the medium takes energy away from each of the propagating partons and rapidly turns that energy into heating of, and collective motion of, the medium itself which is to say extra soft particles in the final state, moving in random directions. This 

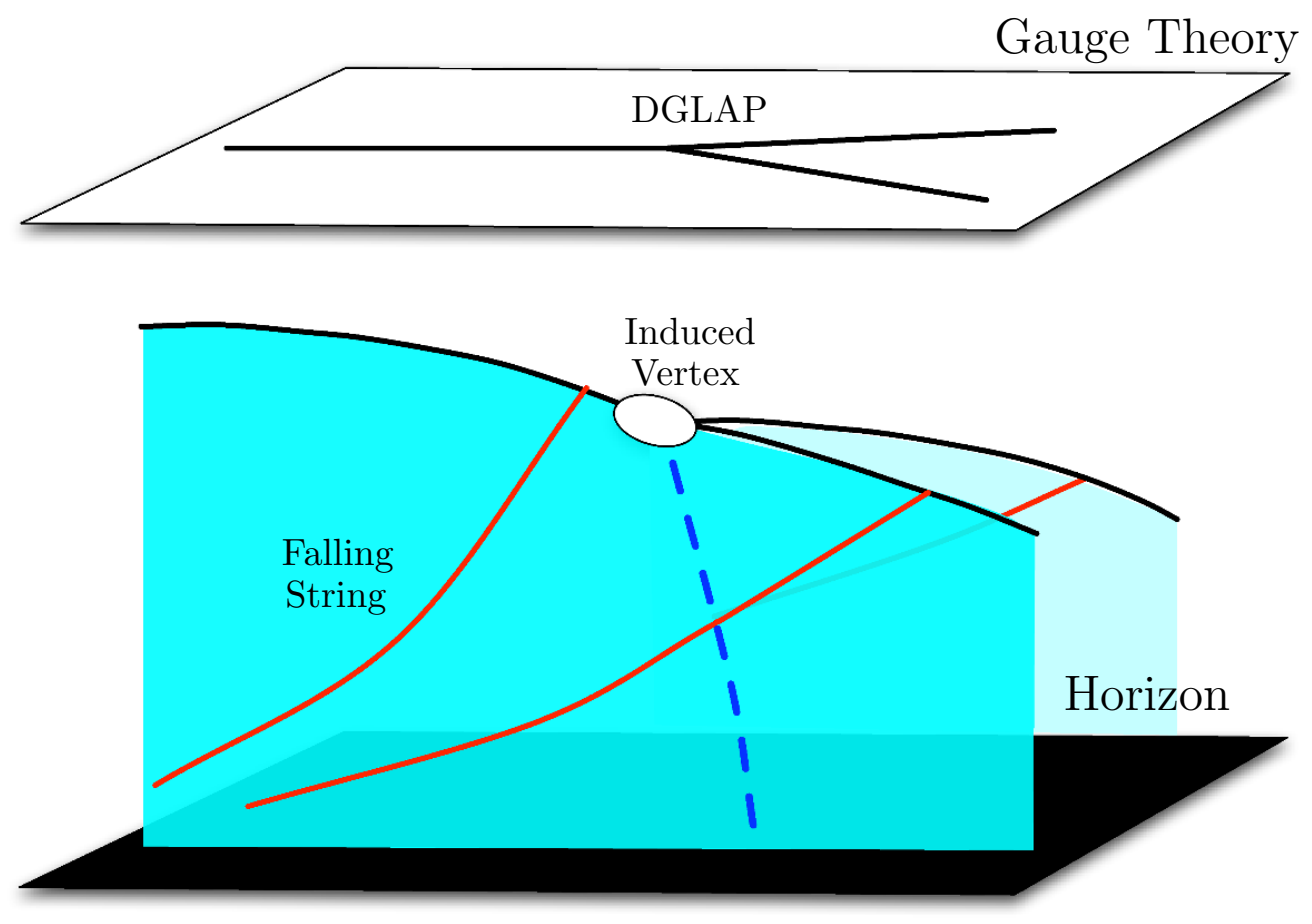

Figure 1. Sketch of two views of the interaction of a high energy jet with the strongly coupled plasma. In the gauge theory, represented by the white plane at the top of the figure, an energetic virtual parton propagates through the medium, loses energy, and splits via (vacuum) DGLAP evolution. There is no (easy) way to describe the strong soft interactions between the partons and the medium in this representation. In the dual gravitational view, represented below, the soft interactions are represented by a string trailing behind each parton, transporting energy from the parton "down" to the horizon which is represented by the black plane at the bottom of the figure. The parton itself, represented by the endpoints of the string which is to say by the black lines, is also pulled "downward" toward the horizon. In this representation, there is no (easy) way to describe the splitting of one string into two, which is to say the hard splitting process in the gauge theory. In the picture we represent the splitting vertex at which one string becomes two by a white oval below which a hypothetical string-splitting curve shown as a blue dashed line trails. At present there is no known calculation underlying these aspects of the figure.

directly yields a reduction in the overall energy of the jet. This is in stark contrast with the perturbative mechanism of radiative energy loss, where energy is lost through medium induced radiation of gluons with momenta that are well above the medium scale and that are typically almost collinear with the initial hard parton when they are produced. This radiative loss of energy by the hard parton translates into a loss of energy for the jet in the final state only if the radiated gluons are either (atypically) produced at large angles relative to the direction of the hard parton or if the radiated gluons are deflected by their further interactions with the medium [33, 34].

In a nutshell, we shall assume that no hard radiative processes occur between the DGLAP vertices and that the dynamics of these partons in the plasma is analogous to that of energetic objects propagating through the strongly coupled plasma in a gauge 
theory with a dual gravitational description. While the theories that possess a gravity dual do not yet include QCD, we will utilize the powerful ideas of the duality to gain qualitative understanding of the relevant strongly coupled dynamics, an approach that has proved useful in many contexts. (See ref. [6] for a review.) However, keeping in mind that these calculations are not done in QCD itself, we will use the explicit results obtained via holographic calculations only as indicative, specifically by keeping all of their parametric dependences while introducing one free dimensionless parameter that we shall fit to data. We shall describe how we do this in concrete terms over the course of sections 3 and 5 .

One important aspect of how we set up our hybrid model is minimalism. We will keep, as much as possible, only well-understood weakly coupled and strongly coupled physics and introduce as few as possible, in fact only one, phenomenological parameter that governs how we put the two together and that needs to be fixed by fitting to data. Introducing further physics into the model would on the one hand allow us to describe some of the less important physics that, as we have described, we are leaving out but on the other hand it would introduce further parameters. Our goal here is to construct a hybrid model that is, in this sense, as simple as we can make it and see how well it does when confronted with data.

The underlying picture that we are putting forward in this paper is sketched in figure 1 . In the gauge theory, any of the partons of the jet which propagate in plasma may suffer a hard splitting, governed by the DGLAP equations. In addition to these hard splittings, these partons possess associated soft fields that interact strongly with the medium. These have a natural interpretation in a dual gravitational representation: they are strings trailing behind the quark, which is represented by the end point of the string. As noted in ref. [22] the string end point itself does not live on the boundary of the spacetime, but "falls" downward, away from the boundary and toward the horizon, as the system evolves. In the gravitational representation, the trailing strings carry energy from the quarks "down" toward the horizon. This represents the process by which each quark loses energy, energy which subsequently thermalizes, making a little more or a little hotter plasma. Reading the figure from left to right, one string enters from the left, with its shape controlled by well-understood gravitational dynamics that describes how the single quark represented by its endpoint loses energy. Next, a perturbative hard splitting, described in the gauge theory by DGLAP, occurs. It is not presently known whether, and if so how, this splitting process can be described in the gravitational representation. The gravitational description must be supplemented by some induced vertex and an associated line along which string world sheets merge, but the form of this vertex and string merging line are not known. Nevertheless, the gauge theory representation demands that after this splitting process, we have two string end points below each of which a string trails. In fact, if one of the daughter partons is a gluon, a double string must be formed, trailing below the corresponding endpoint. So, somehow, the single incident string worldsheet splits into the several world sheets that describe the decay products of the DGLAP splitting. Again, we describe this process in the gauge theory because it is not known how to describe it gravitationally.

Since splitting processes happen at short distances, the induced vertex must occur first as close as possible to the boundary, i.e. where the string end point splits, and only later propagate toward the horizon as represented in figure 1 by the blue dashed line. This 
can also be interpreted as a delay in the ability of softer modes to resolve the splitting of color charges. Nevertheless, since the geodesic distance in AdS from the horizon to the boundary is finite, of order $1 /(\pi T)$, after this short time the string world-sheet is fully split and each of the objects propagate independently through the strongly coupled plasma. ${ }^{1}$ After the transient behavior associated with the splitting, the energy loss of each of the daughter partons in the strongly coupled plasma is described by the dynamics of their own trailing string - until each of the daughters itself splits at a new hard vertex, and the process iterates. We are far from providing a firm theoretical footing for the hybrid physical picture we have described. Each half of the hybrid is built upon solid ground, but different solid ground. In this paper, we will explore the phenomenological consequences of these ideas in a simplified model implementation which we hope captures the main features of some future complete computation.

\section{In-medium energy loss of energetic particles}

The principal ingredient that remains to be specified in the description of our hybrid model is the rate of energy loss of energetic particles in the medium. In our model we shall apply such a prescription to each of the partons in a shower, while those partons find themselves in a medium with local temperature $T$, with $T$ varying as a function of space and time. In this section, we specify the different prescriptions for energy loss that we have investigated by giving them for the case of a single energetic parton propagating through a medium with constant temperature $T$. Our principal goal is of course to investigate the validity of the hybrid strong/weak coupling approach to jet quenching that we have described, in which the shower develops according to a weakly coupled prescription and each parton in it loses energy according to a strongly coupled prescription. However, to provide benchmarks for our computations we shall also try employing weakly coupled prescriptions for how each parton in the shower loses energy in our formalism and compare results obtained in this way to the results we obtain in our hybrid strong/weak coupling model. In the two subsections below we specify the details of the strongly coupled and weakly coupled expressions for parton energy loss that we shall employ.

\subsection{Parton energy loss at strong coupling from falling semiclassical strings}

The problem of energy loss of energetic light degrees of freedom in strongly coupled gauge theories with a gravity dual has been studied extensively. (See refs. [22-25, 37-45] for entries into the literature.) These studies can be divided into two general classes: those in which a hard process in a strongly coupled gauge theory is studied via the gauge/gravity correspondence, for example via analyzing the decay of a virtual external U(1) field into strongly coupled matter within the plasma $[24,25,39,41,42]$; and those in which single

\footnotetext{
${ }^{1}$ Here, we are describing a delay of order $1 /(\pi T)$ in the response of the energy loss process to a sudden change in the nature of the object losing energy, namely the splitting process. In ref. [35] a similar delay time, also of order $1 /(\pi T)$, arises (and is analyzed quantitatively) in the case where the object losing energy (a heavy quark being dragged at constant velocity) is unchanged but the temperature of the strongly coupled medium changes suddenly.
} 
energetic excitations are described as a string moving in the dual gravitational spacetime whose endpoint is attached to a space-filling D7-brane and can therefore fall into the horizon $[22,23,45]$. The former has the advantage that the set-up is fully determined within the strongly coupled theory, while in the latter the initial conditions that characterize the hard creation of these excitations need to be specified. The latter has the advantage that the string describes an isolated excitation whose energy can be tracked, emerging from the initial configuration. These two approaches lead to qualitatively similar results for certain observables, such as the parametric dependence of the maximal stopping distance of energetic partons, but differ quantitatively. While both computations are valid within the context of strongly coupled gauge theories, it is unclear which is a better proxy for QCD hard processes in strongly coupled medium. Since the string-based computations provide the energy loss rate explicitly [45], we will adopt this second approach to construct our hybrid model.

In refs. [22, 45], a pair of high energy 'quark jets' in the fundamental representation of the gauge group are produced moving in opposite directions. In ref. [45] the setup is such that one of the 'quark jets' is incident upon a 'slab' of strongly coupled plasma with temperature $T$, that is finite in extent with thickness $x$. The dual gravitational description of the 'quark jet' is provided via a string whose endpoint falls downward into the bulk, as in the left portion of the sketch in figure 1. After propagating for a distance $x$ through the plasma the string, which is to say the quark, emerges into vacuum. The energy $E$ of the 'quark jet' that emerges from the slab of plasma, as well as its other properties, can be compared to the initial energy $E_{\text {in }}$ of the parton incident upon the slab and to the properties of the 'jet' that would have been obtained had their been no slab of plasma present [45]. For our purposes, we are interested in how the energy of the 'quark jet' depends on $x$, which is to say the rate of energy loss $d E / d x$. If the high energy 'quark' is produced next to the slab, meaning that it enters it immediately without first propagating in vacuum, and if the thickness of the slab is large enough that initial transients can be neglected, meaning $x \gg 1 /(\pi T)$, the rate of energy loss is independent of many details of the string configuration and takes the form [45]

$$
\frac{1}{E_{\text {in }}} \frac{d E}{d x}=-\frac{4}{\pi} \frac{x^{2}}{x_{\text {stop }}^{2}} \frac{1}{\sqrt{x_{\text {stop }}^{2}-x^{2}}}
$$

where $E_{\text {in }}$ is the initial energy of the 'quark', as it is produced and as it is incident upon the slab of plasma and where $x_{\text {stop }}$ is the stopping distance of the 'quark'. Since $E \rightarrow 0$

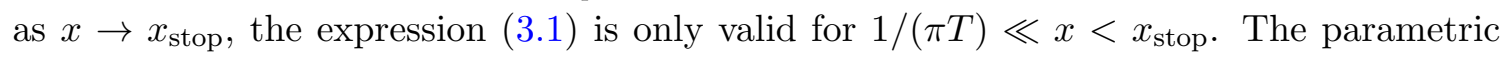
dependence of $x_{\text {stop }}$ on $E_{\text {in }}$ and $T$ was obtained previously in refs. [22, 23]. For a string whose initial state is prepared in such a way as to yield the maximal stopping distance for a 'quark' produced with a given $E_{\text {in }}$ propagating through the strongly coupled $\mathcal{N}=4$ SYM plasma with temperature $T$, it is given by

$$
x_{\text {stop }}=\frac{1}{2 \kappa_{\mathrm{sc}}} \frac{E_{\mathrm{in}}^{1 / 3}}{T^{4 / 3}},
$$

where we have introduced a dimensionless constant $\kappa_{\mathrm{sc}}$, the subscript signifying "Strong Coupling", that in the calculation of ref. [22] is given by $\kappa_{\mathrm{sc}}=1.05 \lambda^{1 / 6}$, with $\lambda$ the 't Hooft 
coupling. In the case of a slab of plasma in which $T$, and therefore $x_{\text {stop }}$ is constant, the energy loss rate (3.1) can easily be integrated to obtain $E(x)$ [45]. We shall be describing the energy loss of partons in a shower that are propagating through a medium whose temperature is changing as a function of space and time as in a heavy ion collision; in this context what we need from ref. [45] is $d E / d x$, namely (3.1).

The energy loss rate eq. (3.1) has two characteristic features that distinguish it parametrically from analogous perturbative expressions that describe the energy loss of a single hard parton propagating through (a slab of) weakly coupled plasma with temperature $T$, expressions that we shall provide in the following subsection. First, while $x$ is not yet comparable to $x_{\text {stop }}$ the rate of energy loss $d E / d x$ is independent of $E_{\text {in }}$ and grows rapidly with $x$, with a characteristic $x^{2}$ dependence. Later, though, once $x$ has become comparable to $x_{\text {stop }}$ we see that $d E / d x$ depends in a nontrivial (i.e. non-power-law) way on both $E_{\text {in }}$ and $x$ and grows rapidly, diverging as $x \rightarrow x_{\text {stop }}$ and $E \rightarrow 0$. We note that in spite of the simple relation between $E_{\text {in }}$ and the stopping distance $x_{\text {stop }}$, the parametric dependence of the energy loss rate on the path length $x$ is intricate, deviating from a simple power of the length very substantially at late times.

The energy lost by the energetic parton propagating through the strongly coupled plasma is quickly converted into hydrodynamic excitations with wave vectors $q \sim \pi T$ and smaller. This happens over a very short time $1 / \Gamma_{1}$, with $\Gamma_{1}=2 \pi T \sim T / 0.16$ the width of the lowest non-hydrodynamical quasinormal mode of the strongly coupled plasma, determined in the dual gravitational theory in ref. [46]. The hydrodynamic excitations are, in turn, dissipated as heat after a damping time $3 T s /\left(4 q^{2} \eta\right)$ (for sound waves) or $T s /\left(q^{2} \eta\right)$ (for diffusive modes) [47]. If we take the shear viscosity to entropy density ratio to be $\eta / s \sim 2 /(4 \pi)$, hydrodynamic modes with $q \sim \pi T$ dissipate over a time $\sim(0.5-0.6) / T$. Longer wavelength modes live longer. This means that most of the 'lost' energy rapidly becomes part of the plasma, thermalizing and resulting in a little more, or a little hotter, plasma. From an experimental point of view, the lost energy becomes extra, soft, hadrons with momenta $\sim \pi T$ moving in random directions. These extra hadrons will be uniformly distributed in angle, on average, if the passage of the jet does not induce any substantial collective motion of the plasma.

Because we shall focus on reconstructed jet data, which is to say measurements of the components of the jet that emerge from the plasma, we shall make no attempt to track the lost energy in our hybrid model. Of course, since the 'lost' energy ends up as soft hadrons going in all directions, some of it will end up in the jet cone. We will make no attempt to add soft hadrons corresponding to some of the lost energy to the jets in our model. The reason that we make no such addition to our jets is that when experimentalists reconstruct jets from data, they use some background subtraction procedure designed to remove soft hadrons that are uncorrelated with the jet direction, for example subtracting an $\eta \leftrightarrow-\eta$ reflection of the event from the real event. This means that if the 'lost' energy ends up perfectly uniformly distributed in angle, it will be subtracted during the jet reconstruction procedure. If this assumption is correct, the 'lost' energy does not appear in the jets as reconstructed by the experimentalists. We therefore make no attempt to add it to the jets we obtain from our model. We leave to future work the investigation of fluctuations and 
collective flow that can in fact result in the 'lost' energy that is deposited in the jet cone not being fully removed during the background subtraction, meaning that some of it ends up being counted as a part of the jet. The uncertainty associated with these considerations means that when we compute jet fragmentation functions in section 5 , they may not be reliable for components of the jet with momenta of order $1-2 \mathrm{GeV}$.

Although the energy loss rate $d E / d x$ in (3.1) was derived within the string-based computation of refs. $[22,45]$, the parametric dependence of the stopping distance for excitations with the maximum possible stopping distance for a given energy $E_{\text {in }}$ given by the expression (3.2) is common to both ways of describing high energy excitations in the plasma discussed above, which makes it seem a robust expectation from strong coupling computations within the gravitational description of large- $N_{c} \mathcal{N}=4 \mathrm{SYM}$ theory. In contrast, the explicit value of the dimensionless constant $\kappa_{\mathrm{sc}}$, and even its dependence on the 't Hooft coupling, are not robust in the same sense. There is every reason to expect that the numerical value of $\kappa_{\mathrm{sc}}$ will be smaller in the strongly coupled QCD plasma than in the strongly coupled $\mathcal{N}=4$ SYM plasma. And, even in the latter theory, the calculations of refs. [24, 25] indicate a value $\kappa_{\mathrm{sc}}$ that is $\mathcal{O}(1)$, i.e. finite in the $\lambda \rightarrow \infty$ limit, rather than $\mathcal{O}\left(\lambda^{1 / 6}\right)$ as in the stringbased calculation of ref. [22]. We shall return to this point in section 6 when we discuss the implications of the value of $\kappa_{\mathrm{sc}}$ that we shall obtain via comparison to data in section 5 .

Both eqs. (3.1) and (3.2) were derived for energetic particles in the fundamental representation of the gauge group, proxies for energetic quarks propagating through the strongly coupled plasma. However, it is impossible to model hard processes in high energy hadronic collisions without also having the means with which to include energetic particles in the adjoint representation, i.e. energetic gluons. In our context, regardless of the identity of the initial parton produced in a hard scattering, the shower of partons that results and whose energy loss we shall be following necessarily includes both gluons and quarks. Studies of high energy particles in the adjoint representation, modeled by double strings propagating through the plasma, were initiated in ref. [23] and have shown that these excitations also have $x_{\text {stop }} \propto E_{\text {in }}^{1 / 3} / T^{4 / 3}$. Within the string-based picture, because the string configuration representing an energetic gluon possesses two strings trailing behind the 'endpoint' (actually, in this case, the point where the string folds back upon itself) it is natural to expect that the stopping distance for a gluon is identical to that for a quark with half the energy of the gluon [22]. We will further interpret this factor 2 as the large $N_{c}$ limit of the ratio of the Casimirs of the adjoint and the fundamental representations of the color gauge group. Given these considerations, we will assume that an energetic gluon has the same energy loss rate eq. (3.1) but with the prefactor in the stopping distance (3.2) given by

$$
\kappa_{\mathrm{sc}}^{G}=\kappa_{\mathrm{sc}}\left(\frac{C_{A}}{C_{F}}\right)^{1 / 3}
$$

with $C_{A} / C_{F}=9 / 4$ the ratio of Casimirs, meaning that $x_{\text {stop }}$ for gluons is shorter than that for quarks with the same energy, but only by a factor of $(9 / 4)^{1 / 3}$.

Because of the small $1 / 3$ power, the difference between the rate of energy loss of quarks and gluons is small, much smaller in the strongly coupled plasma than would be the case 
in a weakly coupled plasma. We will elaborate on the consequences of this observation in section 6.4.

\subsection{Comparison with other approaches}

The realization that the physics at the medium scale is not weakly coupled has motivated several previous phenomenological attempts to implement strongly coupled computations of the in-medium interaction of high energy particles in the modeling of hard processes in heavy ion collisions. Before we continue, it is important to compare and contrast our implementation to those in previous work.

Some early explorations were based on the straightforward use of energy loss rates based upon results derived for a single heavy or light quark traversing the strongly coupled plasma of a gauge theory with a holographic description [29-31, 44, 48-50]. These computations are all aimed at describing the suppression of the production of a single high- $p_{\mathrm{T}}$ hadron, i.e. the leading hadron in a jet. None of these early explorations included the calculation of jet observables; we shall analyze three complementary classes of jet observables in section 5. These early explorations also do not include the perturbative QCD evolution of the hard virtual parton. And, as they describe single partons, they cannot address the question of how the propagation through the strongly coupled plasma does or does not modify the jet fragmentation function, a question that we shall find plays a significant role in differentiating between energy loss mechanisms. Furthermore, in some cases [29, 31, 49] the rate of energy loss of a hard parton is assumed to be a power law in the parton energy and the propagation distance, whereas we now know from ref. [45] that this is true only for partons which do not travel a significant fraction of their stopping distance, as for those and only those partons $d E / d x \propto E_{\text {in }}^{0} x^{2}$. The complete dependence of $d E / d x$ in (3.1) on $x$ and $E_{\text {in }}$ is very different from a power law. In other cases [30], the energy loss rate employed was based on approximations to the numerical analysis of ref. [40], which do not coincide in any limit with the expression derived in ref. [45]. The energy loss expressions obtained more recently in ref. [44] are complementary, in that they are derived in the dual gravitational theory using semiclassical strings that do not satisfy standard open string boundary conditions, meaning that it remains to be determined how they can be used in the description of light quark energy loss.

Among the work that comes before ours, the study that is in many respects most similar to ours is that described in ref. [28], although like in the previous work above this study focusses on hadronic observables rather than computing jet observables as we do. Unlike in the previous work above, this study involves a Monte Carlo implementation of a shower in which partons produced at high virtualities evolve down to a hadronic scale. However, the implementation of the strongly coupled dynamics used in ref. [28] is very different than our own, as it is based on an early interpretation of strongly coupled energy loss in partoniclike terms advocated in refs. [37, 51]. In this approach, the energy loss of a hard parton in strongly coupled plasma is interpreted in the language of radiative energy loss, except with a momentum transfer from the plasma which grows linearly with propagation distance. (In the standard weakly coupled perturbative analysis of radiative energy loss, it is the square of the momentum transfer which grows linearly with propagation distance.) Based 
upon this earlier work, the authors of ref. [28] assumed an energy loss mechanism in which weakly coupled high momentum gluons are radiated (as at weak coupling) but in which the momentum transverse to the jet direction that is transferred to the radiated gluons accumulates linearly with propagation distance (unlike at weak coupling). So, although we follow ref. [28] in the sense that we are developing a hybrid model that melds together features of energy loss in a strongly coupled gauge theory with a Monte Carlo (in our case PYTHIA) implementation of perturbative splitting in a parton shower, our implementation of the strongly coupled physics is completely different than that in ref. [28], since we (i) treat all strongly coupled processes as occurring at soft, nonperturbative, scales; (ii) use the energy loss rate derived from a complete strong coupling computation that was not yet available at the time of the study in ref. [28]; and (iii) incorporate a feature that is by now understood to be characteristic of energy loss in a strongly coupled plasma, namely that the 'lost' energy becomes extra heat or extra plasma, which is to say soft particles whose directions are uncorrelated with the jet direction.

\subsection{Perturbative benchmarks: radiative and collisional energy loss in a weakly coupled plasma}

To gauge the sensitivity of the classes of jet measurements that we will use to constrain our hybrid approach, we wish to compare its results to those in which we replace the strongly coupled result (3.1) for the energy loss rate of a parton in the shower with a perturbatively inspired expression for $d E / d x$. We shall in fact use two different variants as benchmarks.

In the high parton energy limit, upon assuming weak coupling between the energetic parton and the medium the dominant mechanism of energy loss is the radiation of nearly collinear gluons from the energetic parton that is induced by interactions between the parton and the medium. If the medium is sufficiently large that many gluons are radiated from the propagating parton, the energy loss rate for a parton in representation $R$ is given, to leading logarithmic accuracy, by [8]

$$
\frac{d E}{d x}=-\alpha_{s} \frac{C_{R}}{2} \hat{q} x
$$

with $\alpha_{s}$ and $C_{R}$ being the strong coupling constant and the Casimir of the parton, and where the jet quenching parameter $\hat{q}$ is the transverse momentum squared picked up by the parton per distance travelled. While the expression (3.4) describes energy loss in the limit in which many gluons are radiated, in most phenomenological applications of radiative energy loss it is assumed that a finite number of hard gluons are emitted from the the energetic partons and eq. (3.4) describes the average over many partons with a fixed energy.

By dimensional analysis, the jet quenching parameter $\hat{q} \propto T^{3}$. For a very weakly coupled plasma at exceedingly high temperatures, temperatures such that leading order, leading logarithm, perturbative computations are trustworthy, the jet quenching parameter is given by [52]

$$
\hat{q}=C_{A} \alpha_{s} m_{D}^{2} T \log B_{\mathrm{rad}},
$$

where $m_{D}^{2}=g^{2} T^{2}\left(2 N_{c}+N_{f}\right) / 6$ is the square of the Debye screening length of weakly coupled quark-gluon plasma with $N_{c}$ colors and $N_{f}$ flavors, and $B_{\text {rad }}$ is a jet-energy-dependent 
regulator that cuts off large momentum transfers to the plasma. A regulator is necessary because in a weakly coupled plasma $\hat{q}$ diverges logarithmically with the jet energy $E$. The precise value of $B_{\text {rad }}$ is not currently known, although some authors estimate it to be $B_{\text {rad }} \approx 1+6 E T / m_{D}^{2}$. (See ref. [52] for an extensive discussion of estimates of the value of $B_{\text {rad }}$ and hence $\hat{q}$ in different approximations.) We shall ignore all logarithms, lumping them into a prefactor that we shall denote by $\kappa_{\text {rad }}$, with the subscript referring to 'Radiative', and write

$$
\frac{d E}{d x}=-\kappa_{\mathrm{rad}} \frac{C_{R}}{C_{F}} T^{3} x,
$$

with $C_{R} / C_{F}=1$ for an energetic quark and $C_{R} / C_{F}=9 / 4$ for an energetic gluon. Although below we shall treat $\kappa_{\text {rad }}$ as a parameter to be fit to data, before we go on we should estimate its value in a weakly coupled plasma using the leading logarithmic order perturbative calculation, which we denote by $\kappa_{\text {rad }}^{\text {pert }}$. Combining eqs. (3.4) and (3.5), we obtain

$$
\kappa_{\mathrm{rad}}^{\text {pert }}=2 \pi C_{F} C_{A}\left(\frac{2 N_{c}+N_{f}}{6}\right) \alpha_{s}^{3} \log B_{\mathrm{rad}} .
$$

For later reference, we may evaluate this expression for typical values of the strong coupling constant $\alpha_{s}=0.2-0.3$, as utilized in fits to the data in ref. [52], obtaining

$$
\kappa_{\text {rad }}^{\text {pert }} \sim(0.3-1.0) \log B_{\text {rad }} \sim(2-6),
$$

where in the second equality we have used the expression for $B_{\text {rad }}$ given above for jets with energy $E=100 \mathrm{GeV}$ in a plasma with temperature $T=300 \mathrm{MeV}$. Note that this logarithm is large, which suggests that, even for the high energy jets at the LHC, leading logarithmic expressions such as eqs. (3.4) and (3.5) are inapplicable and a resummation, as advocated in refs. [53-55], may be needed.

The expression (3.6) captures the leading $x$ and $T$ dependence of radiative energy loss at weak coupling. We shall treat $\kappa_{\text {rad }}$ as a free parameter, fitting it to one piece of experimental data and then asking how a model in which we use the expression (3.6) to describe the energy loss of the partons in a shower fares in comparison to other data.

One reason why it makes sense to treat $\kappa_{\text {rad }}$ as a parameter to be fit to data is that not all of the energy radiated from the initial parton corresponds to jet energy loss. At emission, the radiated gluons are nearly collinear with the energetic parton, meaning that if the gluons are energetic enough they remain part of the jet. This corresponds to medium modification of the branching probability within the shower, without significant energy loss from the jet cone. However, the subsequent rescattering and further splitting of the radiated gluons can serve to rapidly soften the gluons, and decorrelate their directions with that of the energetic parton. This decorrelation between the directions of the radiated gluons and the jet direction is expected to be most efficient for the softer radiated gluons and less efficient for the harder radiated gluons [33]. What this means is that the $\kappa_{\text {rad }}$ that we need should be smaller than that obtained in the perturbative calculations, smaller by a factor that is at present hard to estimate.

Note that we do not propose our simplified approach as a competitor to more sophisticated Monte Carlo methods for analyzing the effects of radiative energy loss on jets being 
developed by others [56-61]. It is in fact clearly inferior, since we do not track the radiated gluons, treating them as 'lost'. This approach makes sense in our hybrid model, where the lost energy rapidly becomes soft thermal radiation. It does not make sense quantitatively here. Our goal is solely to have a benchmark against which to compare our hybrid model.

Finally, and with the aim of exploring the sensitivity of different observables to the path-length dependence of the jet energy loss, we will study a somewhat more extreme model for energy loss at weak coupling in which we assume that $d E / d x$ is given by a collisional rate. Collisional energy loss is subdominant to radiative energy loss at weak coupling in the high parton energy limit, and for this reason it is neglected in many studies. However, it has been pointed out [17] that, while subdominant, these processes play an important role, especially for heavy quarks moving through the plasma. Here, we shall not advocate any underlying dynamical picture on the basis of which to justify including collisional processes. What we shall do, simply, is to introduce a third model in which, like in our hybrid model, parton branching within the shower proceeds as in vacuum and in which the energy loss of each parton in the shower is given by the collisional energy loss rate in a weakly coupled plasma, whose parametric dependence takes the form [17]

$$
\frac{d E}{d x}=-\kappa_{\mathrm{coll}} \frac{C_{R}}{C_{F}} T^{2}
$$

where we treat $\kappa_{\text {coll }}$ (this time the subscript signifies "Collisional") as a fit parameter to be constrained by one piece of experimental data. This expression captures the leading temperature, energy and path length dependence of the perturbative collisional rate. For an ultra-relativistic parton in a weakly coupled plasma, $\kappa_{\text {coll }}$ is given to leading logarithmic order in perturbation theory by [17]

$$
\kappa_{\text {coll }}^{\text {pert }}=C_{F} \pi \alpha_{s}^{2}\left(\frac{2 N_{c}+N_{f}}{6}\right) \log B_{\text {coll }} .
$$

where, as before, $B_{\text {coll }}$ regulates the effect of large momentum transfer scatterings in the medium and is understood to be proportional to the parton energy. The precise expression for $B_{\text {coll }}$ depends on the criteria used in the regularization; see ref. [17] for a compilation of expressions from the literature. As in the case of radiative energy loss, we can substitute $\alpha_{s}=0.2-0.3$ into (3.10) and estimate the value of $\kappa_{\text {coll }}$ if we assume that these values of $\alpha_{s}$ are small enough for a leading logarithmic calculation to be relevant, obtaining

$$
\kappa_{\text {coll }}{ }^{\text {pert }} \sim(0.25-0.6) \log B_{\text {coll }} \sim 1.6-3.3,
$$

where in the second equality, we have used $B_{\text {coll }}=6 E T / m_{D}^{2}$. As in the case of radiative energy loss, the logarithmic factor is large which means that it is doubtful that these values of $\alpha_{s}$ are small enough for these leading logarithmic expressions to be reliable.

We have chosen the ratio of Casimirs appearing in both eqs. (3.6) and (3.9) such that the parameter $\kappa_{\text {rad }}$ (or $\kappa_{\text {coll }}$ ) that we shall obtain by fitting our expressions for the radiative (or collisional) energy loss to data is that for the energy loss of a quark moving through a weakly coupled plasma, while a gluon gets an additional factor. Note that the dependence of eqs. (3.6) and (3.9) on $C_{A} / C_{F}$ is much stronger than that in (3.1), obtained at strong 
coupling. We will return to this important distinction between energy loss in a strongly coupled plasma and that in a weakly coupled plasma in section 6.4.

\section{Monte Carlo implementation}

The implementation of the hybrid model that we have described requires several steps, beginning with the generation of jets and the modification of their evolution due to energy loss, but also including the hydrodynamic calculation of the space and time dependence of the bulk medium created in the heavy ion collision. The procedures used for the calculations reported in this work are presented in this section.

We generate hard processes using PYTHIA 8.170 [62]. ${ }^{2}$ Since at the LHC center of mass energy and in the range of momentum relevant for our analysis $\left(p_{\mathrm{T}} \sim \mathcal{O}(100 \mathrm{GeV})\right)$, the modification of the nuclear parton distribution functions with respect to the proton ones is very small $[63,64]$, we simulated high energy jet production in proton-proton collisions at $\sqrt{s}=2.76 \mathrm{TeV}$. Since these events are later embedded into a hydrodynamic model for the bulk matter produced in the nucleus-nucleus collisions, we do not include the underlying event in the PYTHIA treatment of the proton-proton collision in our calculation. We use the PYTHIA $p_{\mathrm{T}}$-ordered shower to evolve the hard process from the initial virtuality down to a typical hadronic scale of $Q_{0}=1 \mathrm{GeV}$, at which we stop the evolution. At this scale, vacuum event generators switch to phenomenological models of hadronization, like the Lund string model which is incorporated into PYTHIA. For a number of reasons, the nonperturbative hadronization process is expected to be altered in a heavy ion collision relative to that in vacuum. For example, most of the soft hadrons in a heavy ion collision will be formed via the coalescence of quarks and gluons from the expanding and cooling plasma rather than directly from partons produced initially and their fragments $[65,66]$. Furthermore, even if we only look at hadrons that are formed via fragmentation, hadronization in this setting is still modified by the presence of the medium via changes in how color flows [67-72]. In order to avoid complicating the interpretation of our results with currently unconstrained hadronization dynamics, throughout this paper we will work at the partonic level and focus on observables that are less sensitive to the hadronization process. For example, in jet observables these corrections are, at least in vacuum, smaller than $10 \%$ [73].

On an event by event basis, the events generated by PYTHIA each initiate a decay chain which will be the starting point for our implementation of medium effects. As we have argued in section 2 , in our hybrid model we shall neglect the possibility that the presence of the medium may result in modification of the splitting probabilities, or modification to the locations in space and time where splitting occurs obtained via eq. (2.1). We are neglecting the fact that the reduction in the available energy due to the loss of energy of a parton in the shower leads to a reduction in the phase space available when that parton subsequently splits. In this exploratory study we will neglect such phase space effects and

\footnotetext{
${ }^{2}$ After most of work presented in this paper was completed we became aware that this version of PYTHIA suffers from a bug which affects the description of hadronization. Since we will work at the partonic level throughout, this bug has no effects on our results. We have explicitly checked this by recomputing some of our results using PYTHIA 8.183.
} 
assume that the overall structure of the decay chain remains the same even after we make the partons in the shower lose energy.

We place the point of origin of each of the dijet processes generated by PYTHIA in the plane transverse to the collision axis at a location selected with a probability proportional to the number of binary collisions at that location in the transverse plane. The showers generated by the dijets proceed in space and time according to eq. (2.1), propagating outward along their (randomly selected) direction of motion. Since the dijet production process is hard, dijets are produced very early $(\tau \sim 1 / Q)$, prior to the proper time at which the plasma produced in the collision hydrodynamizes, $\tau_{\text {hydro }}$. We will assume that during the short proper time before $\tau_{\text {hydro }}$, the jets propagate unperturbed. ${ }^{3}$ After $\tau_{\text {hydro }}$, the jets encounter the hydrodynamically expanding plasma and the different fragments of the jet suffer energy loss, according to (3.1) in our hybrid strong/weak coupling model or according to (3.6) or (3.9) in our models of weakly coupled radiative or collisional energy loss. To determine the local properties of the plasma at the position of the fragments, we embed the jet shower into the boost-invariant ideal hydrodynamic simulations of the expanding cooling plasma produced in heavy ion collisions with $\sqrt{s}=2.76 \mathrm{TeV}$ per nucleon that we have obtained from ref. [74]. These simulations reproduce the multiplicity of charged particles produced at mid-rapidity at the LHC. ${ }^{4}$ Since in the simulations of ref. [74] the hydrodynamic fields are initialized at $\tau_{\text {hydro }}=0.6 \mathrm{fm}$, we will take this as our hydrodynamization time. From these simulations we determine the temperature of the plasma at each point in space and time, and hence the spacetime-dependent temperature that each parton in the fragmenting shower encounters on its way through and eventually out of the expanding, cooling, droplet of plasma. We use this spacetime-dependent temperature to integrate the different expressions for the energy loss rate $d E / d x$ discussed in section 3 over the path of each parton in the shower during its lifetime, i.e. from the time when it is produced in a splitting process to the time when it itself splits.

The procedure described above assigns an energy loss to each of the virtual partons in the shower. However, it does not determine how the lost energy is distributed among the several particles that are produced when each virtual parton splits, or decays. Consistent with the assumption that the medium does not change the splitting probabilities in the shower, since these splitting probabilities depend on the energies of the daughter partons only through the fraction of the parent parton energy that each daughter obtains as a result of the splitting we choose to distribute the energy lost by the parent parton as a reduction

\footnotetext{
${ }^{3}$ This is an assumption that could be improved upon in future, once the analysis of the early preequilibrium energy loss of heavy quarks in ref. [35] is extended to light quarks. That analysis indicates that energy loss sets in only after a delay time of order $1 /(\pi T)$ after the moment during the collision when the energy density is at its maximum, $T$ being the temperature at the time of hydrodynamization. In addition, the analysis of the collision of sheets of energy density in ref. [36] indicates that if the sheets are thin enough there is a prior delay of order $1 /(\pi T)$ between the collision time and the time when the energy density peaks. The results of refs. $[35,36]$ together indicate that there will surely be some energy loss before $\tau_{\text {hydro }}$ but that it is not expected to be large.

${ }^{4}$ It would be interesting to repeat our analysis using a three-dimensional viscous hydrodynamics simulation, ideally one that includes event-by-event fluctuations in the initial state at the time of hydrodynamization. We leave this to future work.
} 
in the initial energy of each of the daughters according to this fraction. As they themselves propagate through the medium subsequently, these decay partons loose additional energy until they split again. Therefore, the total energy lost by a particular final parton that escapes from the medium depends on the detailed history of splitting and propagation that led to that parton.

Since the goal of this work is to study the effect on high energy jets of energy loss in strongly coupled plasmas, we will not describe the degradation of the jet energy in the hadron gas produced after the plasma cools through the QCD phase transition at $T \sim T_{c}$. We focus only on the energy loss as the jet propagates through the strongly coupled plasma with $T>T_{c}$. To ensure that we do not apply the strong coupling results to the late time resonance gas, we will stop the computation of energy loss when the temperature of the system falls below $T_{c}$, which we identify with the crossover temperature of the QCD transition that separates the plasma from the hadron gas. Since the QCD transition is a cross-over, $T_{c}$ is not sharply defined and its precise value depends on the procedure used to determine it. The hydrodynamic simulations from ref. [74] that we are using employ an equation of state obtained from the lattice QCD calculations in ref. [75]. Although more recent lattice calculations favor a slightly lower value of $T_{c}$, since we are obtaining the temperature profile from hydrodynamic calculations done according to the QCD thermodynamics of ref. [75] we will vary $T_{c}$ in the range $180<T_{c}<200 \mathrm{MeV}$ specified in ref. [75]. We shall employ this variation in our choice of $T_{c}$ as a device with which to estimate the systematic uncertainty in the results that we obtain from the computations that we shall perform using our hybrid model.

Finally, in addressing RHIC data we will employ an identical procedure except that we start with hard dijets produced (by PYTHIA) in collisions with $\sqrt{s}=200 \mathrm{GeV}$ per nucleon and we replace the hydrodynamic profile for LHC collisions with that for RHIC collisions, also obtained from ref. [74].

In the next section, we describe how we reconstruct the jets in our hybrid model and compare them, in various ways using various measured observables, to jets reconstructed from heavy ion collision data.

\section{Comparison with jet data}

We have described the implementation of our hybrid model in full detail in the two previous sections. All that remains is to choose the one dimensionless free parameter $\kappa_{\text {sc }}$, defined in eq. (3.2), that we have introduced into our description of the energy loss of an individual parton in the PYTHIA shower as it propagates through the strongly coupled plasma and the model will then be fully specified. As explained in section 3, we are assuming that the strongly coupled dynamics fixes the parametric dependence of the energy loss rate $d E / d x$, given in eq. (3.1), and the stopping distance $x_{\text {stop }}$, given in eq. (3.2), but not the overall normalization of $x_{\text {stop }}$. Therefore, our model possesses one free parameter, which we need to fit to data. Once this has been done, we will be able to study different jet observables and extract the effect of the medium on each of them. 


\subsection{Jet reconstruction and jet $\boldsymbol{R}_{\mathrm{AA}}$}

The first observable that we shall compute is $R_{\mathrm{AA}}$ for jets, as a function of $p_{T}$, the transverse momentum of the jet, and as a function of the centrality of the heavy ion collision. ${ }^{5}$ The jet $R_{\mathrm{AA}}$ is the ratio of the number of reconstructed jets with a given $p_{T}$ that we find in heavy ion collisions in a given centrality bin to the number of jets with that same $p_{T}$ in $N_{\text {binary }}$ proton-proton collisions with the same $p_{T}$, where $N_{\text {binary }}$ is the number of protonproton collisions that occur in a heavy ion collision of the given centrality, according to a Glauber model. Because the production cross-section for jets is a rapidly falling function of $p_{T}$, if the jets in a heavy ion collision have lost energy due to the passage of the partons in the jet through the strongly coupled medium this results in $R_{\mathrm{AA}}<1$. To determine the prediction of our model for the jet $R_{\mathrm{AA}}$, we need to reconstruct jets both in heavy ion collisions within our model (as described in previous sections, including the effects of energy loss) and in proton-proton collisions as described by PYTHIA with the underlying event switched off, as explained in section 4. To obtain the principal results of this paper, we generated 300,000 PYTHIA events with $p_{T}$ greater than a cut that we set to $50 \mathrm{GeV}$ for collisions with centralities in each of four ranges (0-10\%, 10-30\%, 30-50\% and 50-70\%). We varied the $p_{T}$ cut to make sure that the jet spectrum in the (higher) range of $p_{T}$ where we performed our analyses was insensitive to the value of the cut. We used the PYTHIA events without modification to describe jets in proton-proton collisions. As described in sections 3 and 4, to describe quenched jets in heavy ion collisions we embed the PYTHIA events in a hydrodynamic description of the matter produced in a heavy ion collision and apply our prescription for energy loss to each parton in the PYTHIA shower. We then analyze the output of our model calculations of quenched jets in heavy ion collisions and of proton-proton jets using FastJet [78], with which we reconstruct jets using the anti- $k_{t}$ algorithm [79]. Defining a jet, via any reconstruction algorithm, requires the specification of a resolution parameter, $R$. This parameter can be understood as the opening angle

\footnotetext{
${ }^{5}$ The "centrality" of a collision between heavy ions refers to its impact parameter. Nearly head-on collisions, with the smallest impact parameters, are referred to as central collisions; peripheral collisions, with large impact parameter, are noncentral. The impact parameter is not directly measured, but it is nevertheless possible to bin heavy ion collision data as a function of impact parameter, for example using the fact that the total number of hadrons produced in a heavy ion collision is anticorrelated with the impact parameter of the collision. Central collisions have the highest multiplicity; peripheral collisions the lowest. Experimentalists therefore bin their events by multiplicity, using that as a proxy for the impact parameter. The terminology used refers, for example, to the " $0-10 \%$ centrality bin" and the " $10-20 \%$ centrality bin", meaning the $10 \%$ of events with the highest multiplicities (and lowest impact parameters) and the next $10 \%$ of events with the next highest multiplicities (and next lowest impact parameters). The correlation between event multiplicity and impact parameter is described well by the Glauber model of multiple scattering [76, 77 ], which relates the event multiplicity to the number of nucleons that participate in the collision $\left(N_{\text {part }}\right)$ which in turn can be related via a geometrical calculation to the impact parameter of the collision. In our calculations, we take the tabulation of the range of impact parameters that corresponds to a given centrality bin defined via the multiplicity distribution for Pb-Pb collisions at the LHC from ref. [74]. When we distribute the points of origin of our PYTHIA jets in the transverse plane, we do so with a probability distribution for the impact parameter $b$ dictated by the number of collisions at each $b$ within the range corresponding to a given centrality bin. In order to then apply our energy loss prescription to the partons in the PYTHIA shower, we embed the PYTHIA jet in the hydrodynamic solution from ref. [74] corresponding to the mean value of the impact parameter in the interval associated with the given centrality bin.
} 

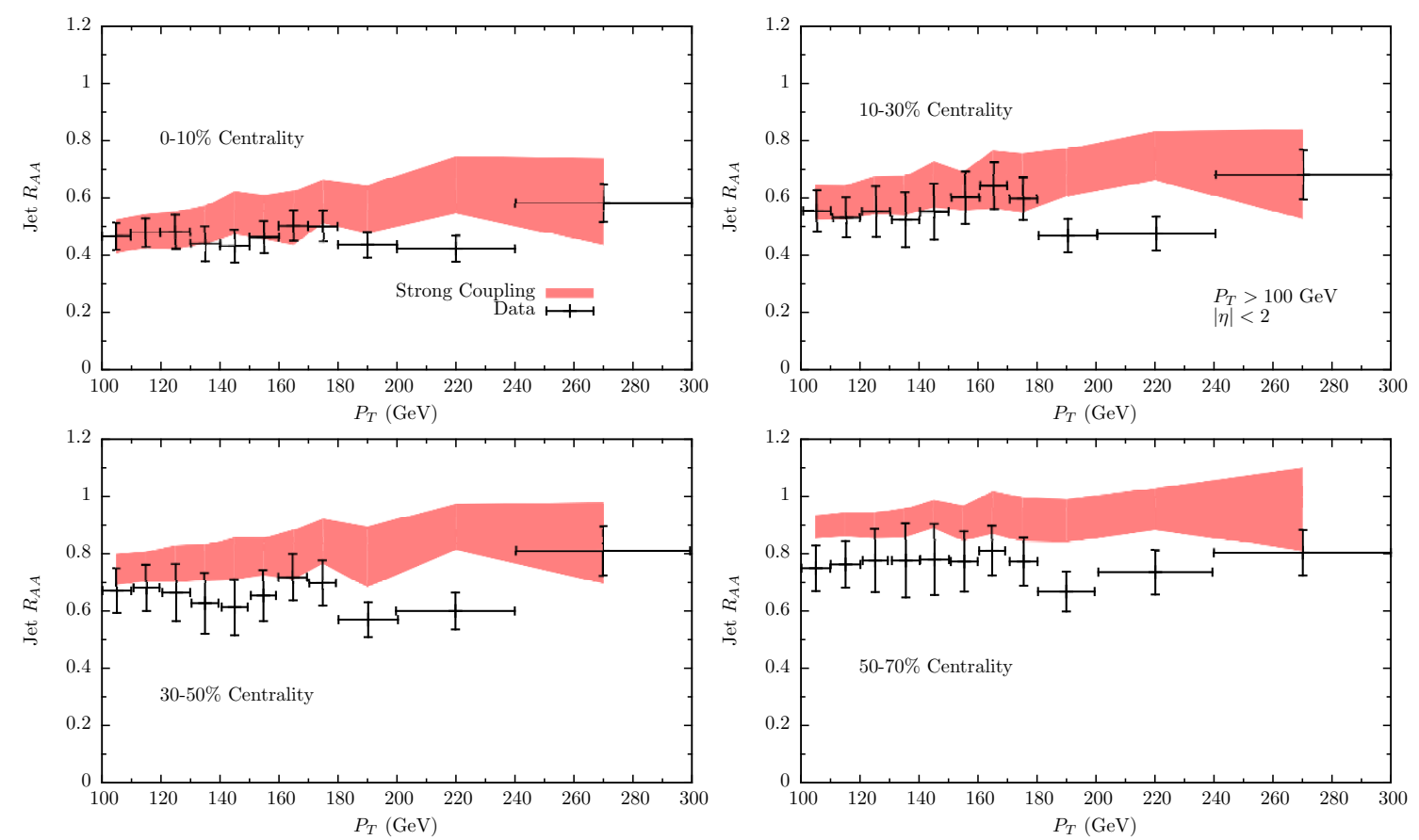

Figure 2. Jet $R_{\mathrm{AA}}$ as a function of $p_{\mathrm{T}}$ for different centralities in our hybrid model for jet quenching in strongly coupled plasma compared to preliminary CMS data from ref. [80]. The results of our calculations in our hybrid strongly coupled model, shown by the colored bands, are completely specified once we have fixed the one free parameter in the model so that the model agrees with the left-most data point in the top-left panel, namely the jets with $100 \mathrm{GeV}<p_{\mathrm{T}}<110 \mathrm{GeV}$ in the most central collisions. Once this point has been fitted, the $p_{\mathrm{T}}$ dependence and centrality dependence of $R_{\mathrm{AA}}$ are outputs of the model.

(in radians) of the jets we reconstruct, although the precise meaning of $R$ is different for different reconstruction algorithms. We shall set the reconstruction parameter in the anti- $k_{t}$ algorithm to $R=0.3$ for $\mathrm{Pb}-\mathrm{Pb}$ collisions at LHC energies and to $R=0.2$ for $\mathrm{Au}-\mathrm{Au}$ collisions at RHIC energies because we shall compare the predictions of our model to jet measurements from LHC and RHIC data that employ these values of $R$. As we have discussed in section 4, the output of our model is partons not hadrons, and we are reconstructing jets from those partons. For this reason, we will focus on jet observables that are relatively insensitive to details of the hadronization process.

In order to fit the value of the one free parameter $\kappa_{\mathrm{sc}}$ in our parametrization (3.1) and (3.2) for the rate of energy loss $d E / d x$ of each of the partons in the PYTHIA shower, we calculate $R_{\mathrm{AA}}$ for jets with $100 \leq p_{T} \leq 110 \mathrm{GeV}$ in the range of pseudorapidities $-2 \leq \eta \leq 2$ in the $0-10 \%$ most central $\mathrm{Pb}-\mathrm{Pb}$ collisions at LHC energies, with collision energy $\sqrt{s}=$ $2.76 \mathrm{TeV}$ per nucleon pair. This quantity has been measured by the CMS collaboration, and in the data it lies between 0.42 and 0.51. (For this and for all experimental data quoted in our paper, we have added the statistical and systematic errors in quadrature.) We find that we can reproduce this measured result with our model as long as we choose $\kappa_{\text {sc }}$ between 0.26 and 0.35 . In determining this range of allowed values of the parameter $\kappa_{\mathrm{sc}}$ we have 

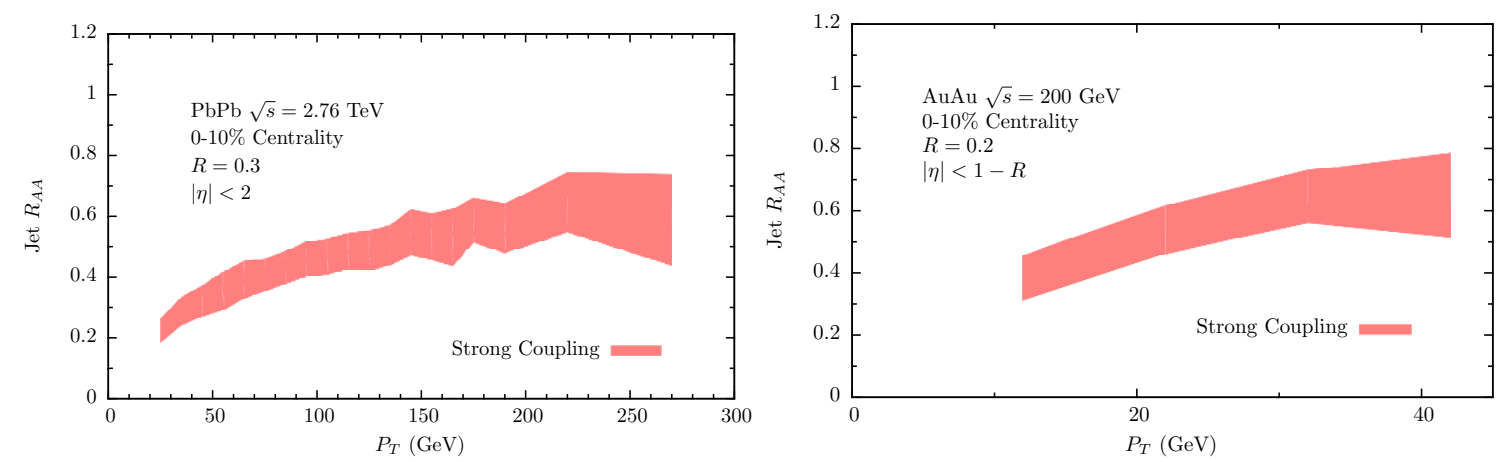

Figure 3. Predictions of our hybrid strongly coupled model for jet $R_{\mathrm{AA}}$ as a function of $p_{\mathrm{T}}$ for central $\mathrm{Pb}-\mathrm{Pb}$ collisions at the LHC with $\sqrt{s}=2.76 \mathrm{TeV}$ per nucleon (left) and Au-Au collisions at RHIC with $\sqrt{s}=200 \mathrm{GeV}$ per nucleon (right). In both cases, we only show our results for collisions in the $0-10 \%$ centrality bin.

included the theoretical uncertainty in the critical temperature $T_{c}$, discussed in section 4 , as well as the uncertainty that enters via the uncertainty in the experimentally measured quantity. The latter dominates the uncertainty in the extracted value of $\kappa_{\mathrm{sc}}$. Henceforth, in all our plots we will show a band of results obtained from our model corresponding to varying $\kappa_{\mathrm{sc}}$ between 0.26 and 0.35 , a range that incorporates both experimental and theoretical uncertainty.

With $\kappa_{\mathrm{sc}}$ now fixed, the first results that we obtain from our model are the dependence of the jet $R_{\mathrm{AA}}$ on $p_{T}$ and on the centrality of the collision, for $\mathrm{Pb}-\mathrm{Pb}$ collisions at $\sqrt{s}=$ $2.76 \mathrm{TeV}$. We show our results in figure 2. We see that our hybrid model predicts a jet $R_{\mathrm{AA}}$ that is only weakly $p_{T}$-dependent, in agreement with the preliminary CMS data from ref. [80]. The evolution of the jet $R_{\mathrm{AA}}$ with increasing centrality is consistent with the data until we get to the most peripheral bin, for which our model predicts less quenching than is seen in the data. This discrepancy may be due in part to the fact that we are not including the energy loss in the hadronic phase in our computation, since peripheral collisions will spend less time in the plasma phase making the time spent in the hadronic phase proportionally more relevant.

In figure 3 we further explore the $p_{\mathrm{T}}$ and $\sqrt{s}$ dependence of the jet $R_{\mathrm{AA}}$ within our hybrid approach. In the left panel, we extend our computation of jet suppression down to $15 \mathrm{GeV}$ for the most central LHC collisions, using a sample of PYTHIA jets generated with $p_{\mathrm{T}}$ greater than a $10 \mathrm{GeV}$ cut. Because the jet production cross-section falls rapidly with $p_{\mathrm{T}}$, in order to have sufficient statistics over this wide range in $p_{\mathrm{T}}$ we generated several independent samples of jets, each with $p_{\mathrm{T}}$ greater than a higher value of the cut than in the sample before, employing cuts of 10,35 and $50 \mathrm{GeV}$. We then merged each sample with the previous one away from these cuts. In this way we were able to obtain a sample of jets with reasonable statistics for $p_{\mathrm{T}}$ ranging all the way from $15 \mathrm{GeV}$ to $270 \mathrm{GeV}$. Even over this extended range of $p_{\mathrm{T}}$, the jet suppression factor $R_{\mathrm{AA}}$ varies relatively little with transverse momentum. This is in qualitative agreement with $R_{\mathrm{CP}}$ measurements by ATLAS [81] and charged jet $R_{\mathrm{CP}}$ measurements by ALICE [82], which both report 

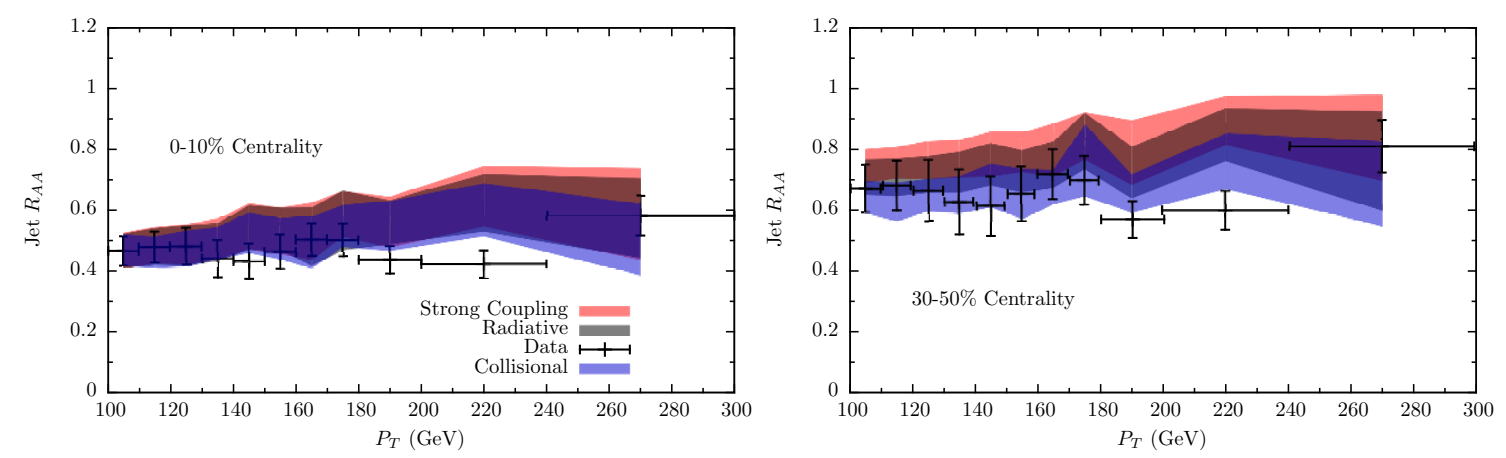

Figure 4. Jet $R_{\mathrm{AA}}$ as a function of $p_{\mathrm{T}}$ for LHC collisions in two different centrality bins for the three energy loss models from section 3, as compared to preliminary CMS data [80]. Each of the three models for the rate of energy loss $d E / d x$ includes one free parameter, and in each case we have fitted the value of this parameter to obtain agreement between the model and the data for $100 \mathrm{GeV}<p_{\mathrm{T}}<110 \mathrm{GeV}$ in the most central $(0-10 \%)$ collisions.

suppression measurements down to this range of $p_{\mathrm{T}}$ with a similarly weak dependence on $p_{\mathrm{T}}$. Nevertheless, at present we refrain from a quantitative comparison with these data, for two reasons. $R_{\mathrm{CP}}$ is the ratio of the number of jets with a given $p_{\mathrm{T}}$ in central collisions to an expectation based upon data in peripheral collisions, rather than an expectation based upon data in proton-proton collisions as in $R_{\mathrm{AA}}$. Given the disagreement that we see between our model and the data in the peripheral bin at the higher values of $p_{\mathrm{T}}$ displayed in figure 2, we cannot make a direct comparison between our results at lower values of $p_{\mathrm{T}}$ in figure 3 and measurements of $R_{\mathrm{CP}}$. And, since we are working at the partonic level, we are at present hesitant to compare our results to measurements of jets defined via charged hadrons only, rather than calorimetrically. In the right panel of figure 3 , we repeat our analysis for the lower jet energies available in RHIC collisions with a center of mass energy of $\sqrt{s}=200 \mathrm{GeV}$ per nucleon, extending our analysis down to $12 \mathrm{GeV}$ using a sample of PYTHIA jets generated with $p_{\mathrm{T}}$ greater than a $5 \mathrm{GeV}$ cut. We chose the jet reconstruction parameter $R=0.2$, as in ref. [83]. Our results are in good agreement with the preliminary experimental measurements reported by the STAR collaboration in ref. [83], at present still with significant systematic uncertainties. However, we have again refrained from making a direct comparison since, as before, it is not easy to compare our partonic jet results with the charged jet measurements reported in ref. [83]. Also, in making these measurements the STAR collaboration requires the presence of a semi-hard $\left(p_{\mathrm{T}}=5-7 \mathrm{GeV}\right)$ charged hadron within the jet, a criterion that is hard for us to reproduce from our partonic computation.

The predictions of our model for both the momentum dependence and the centrality dependence of jet suppression are in encouraging agreement with experimental data. To avoid over-interpreting this agreement, it is important to assess the sensitivity of the jet $R_{\mathrm{AA}}$ observable to the underlying dynamics of the energy loss. To gauge this sensitivity, we have repeated the analysis for the two other models of the energy loss rate $d E / d x$ described in section 3. In figure 4 we show the jet suppression factor $R_{\mathrm{AA}}$ in two centrality bins for the strongly coupled (red), radiative (grey) and collisional (blue) energy loss models. In all three models, as in figure 2 we have fitted the one free parameter in our description of $d E / d x$ 

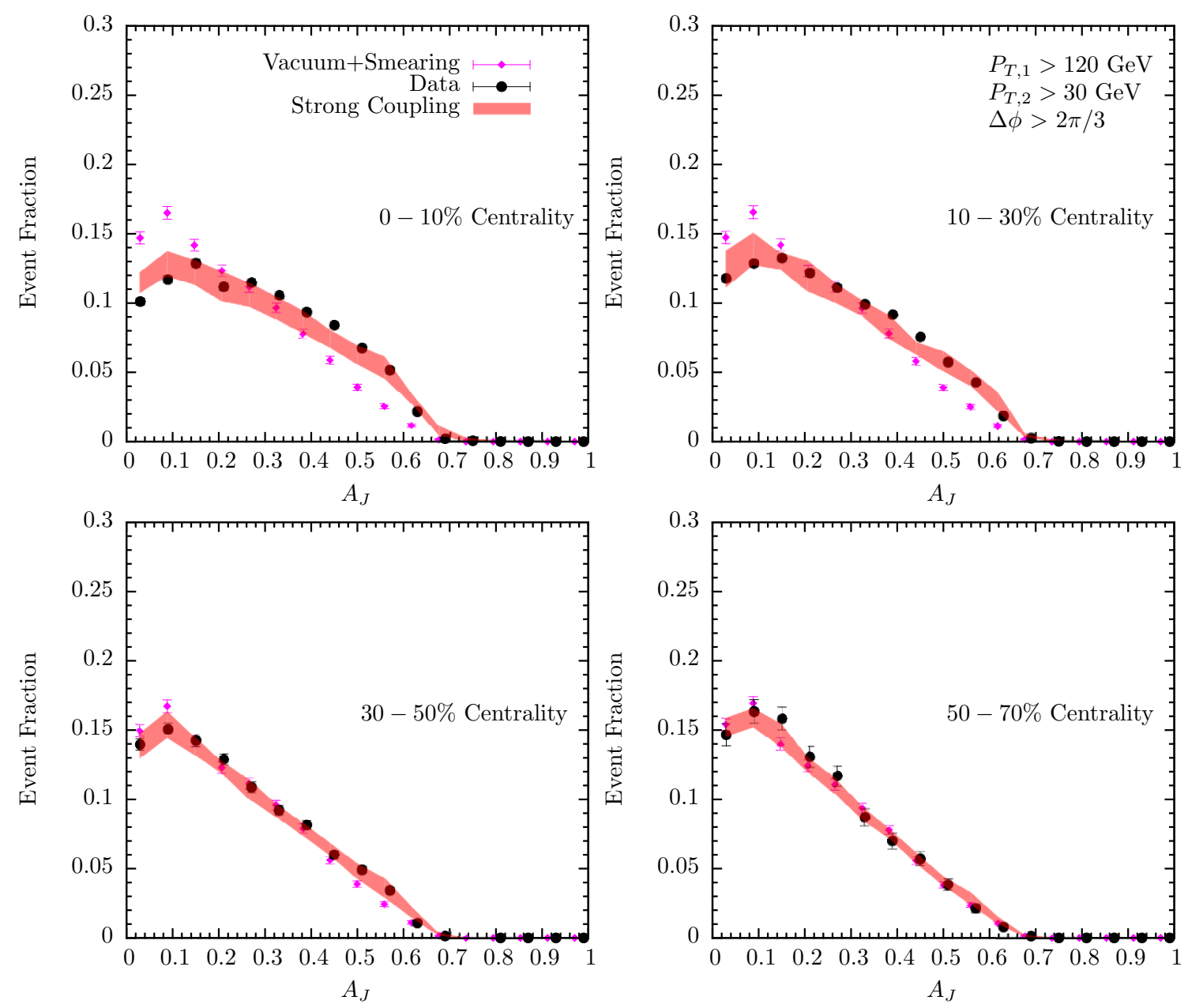

Figure 5. Red bands show the probability distributions for the dijet imbalance $A_{J}$ in LHC collisions with four different ranges of centrality predicted by our strongly coupled hybrid model. The jet momenta are smeared, according to the prescription given in ref. [85] in order to mimic background subtraction effects. Experimental data points are taken from ref. [84]. As a comparison, we show the distributions of the dijet imbalance $A_{J}$ in the proton-proton collisions that we have obtained from PYTHIA, including the (centrality dependent) momentum smearing needed in order to make a fair comparison to the heavy ion results.

to the left-most data point in the left panel, finding $0.81<\kappa_{\mathrm{rad}}<1.60$ for the parameter $\kappa_{\text {rad }}$ defined in the expression (3.6) for $d E / d x$ in our model for weakly coupled radiative energy loss and $2.5<\kappa_{\text {coll }}<4.2$ for the parameter $\kappa_{\text {coll }}$ defined in the expression (3.9) for $d E / d x$ in our model for weakly coupled collisional energy loss. Remarkably, despite the fact that the energy dependence and the path-length dependence of the three different expressions (3.1), (3.6) and (3.9) are very different for the three quite different energy loss mechanisms that we are modelling, the $p_{\mathrm{T}}$ dependence and the centrality dependence of the jet $R_{\mathrm{AA}}$ are quite similar in all three models. 

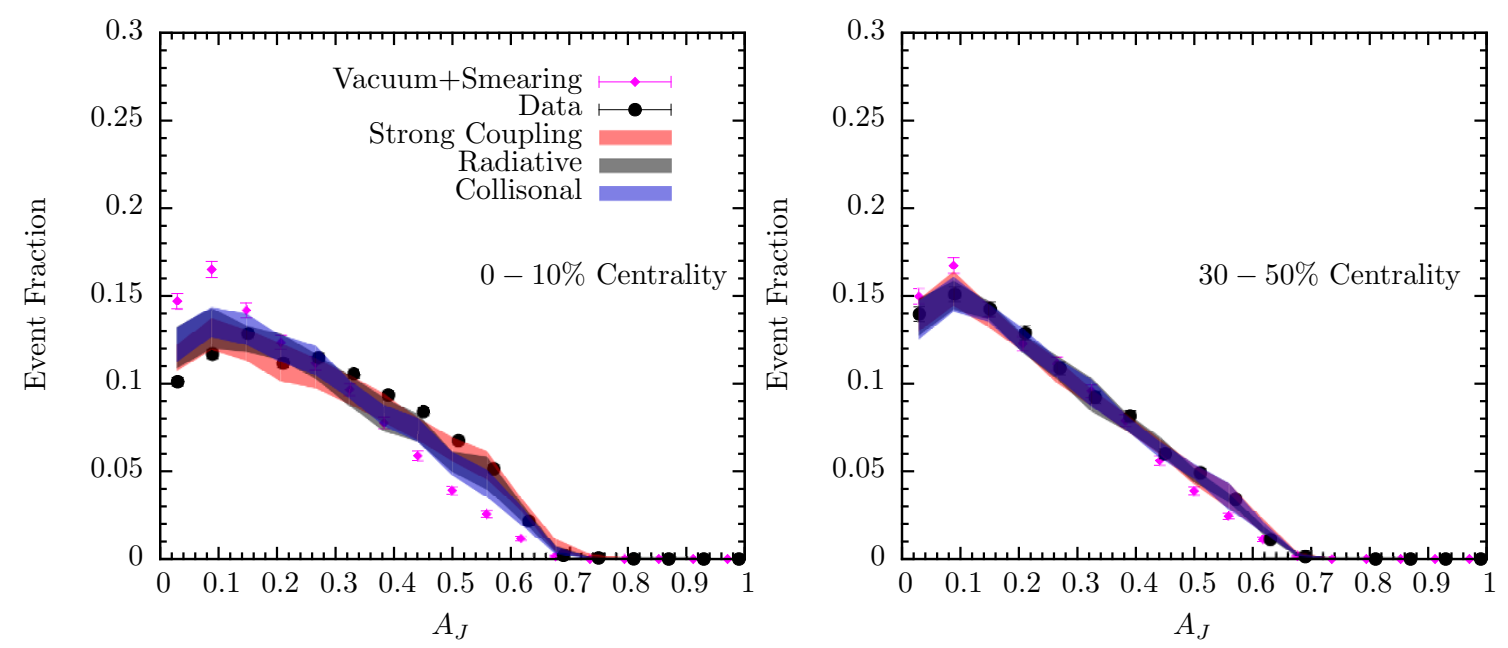

Figure 6. Probability distribution for the smeared dijet imbalance $A_{J}$ for three different models of the rate of energy loss $d E / d x$ in LHC heavy ion collisions in two different ranges of centrality.

\subsection{Dijet asymmetry}

After constraining and then confronting the three models with data on the jet suppression $R_{\mathrm{AA}}$, we turn now to a different jet observable, the dijet imbalance $A_{J}[1,2]$. Following the data analysis procedure used in the analysis of the experimental data reported in ref. [84], in our Monte Carlo simulation we select events containing dijet pairs reconstructed with the anti- $k_{t}$ algorithm with jet reconstruction parameter $R=0.3$ in the pseudorapidity range $|\eta|<2$ such that the leading jet has $p_{\mathrm{T} 1}>120 \mathrm{GeV}$ and the subleading jet has $p_{\mathrm{T} 2}>30 \mathrm{GeV}$. The asymmetry variable is then defined as $A_{\mathrm{J}} \equiv\left(p_{\mathrm{T} 1}-p_{\mathrm{T} 2}\right) /\left(p_{\mathrm{T} 1}+p_{\mathrm{T} 2}\right)$. Since the data presented by both ATLAS [1] and CMS $[2,84]$ for this observable are not fully unfolded from resolution effects, a direct comparison of the result of our computations with data is not possible. However, the CMS collaboration has demonstrated that a simple centrality and momentum dependent smearing procedure can reproduce the systematics of such effects, at least for $\gamma$-jet observables, and has provided an explicit parameterization for such smearing in that type of measurement [85]. Since the corresponding parameterization for dijet measurements is not yet available, we will use the procedure advocated in ref. [85] also for dijets. The result of these computations is a prediction from our strongly coupled hybrid model for the probability distribution for $A_{J}$ for heavy ion collisions at the LHC with four different centrality bins shown in figure 5 . The centrality dependence of the smearing function is illustrated by the violet points which show the results of applying the (centrality dependent) smearing to proton-proton events from PYTHIA. The energy loss experienced by both jets in the dijet pair tends to increase $A_{J}$ in heavy ion collisions, more so in more central collisions. We see this in figure 5 as the widening of the asymmetry distribution in more central collisions, both in the predictions of our model and in the data. We see from the figure that there is good agreement between the predictions of our model and measurements made using LHC data. 

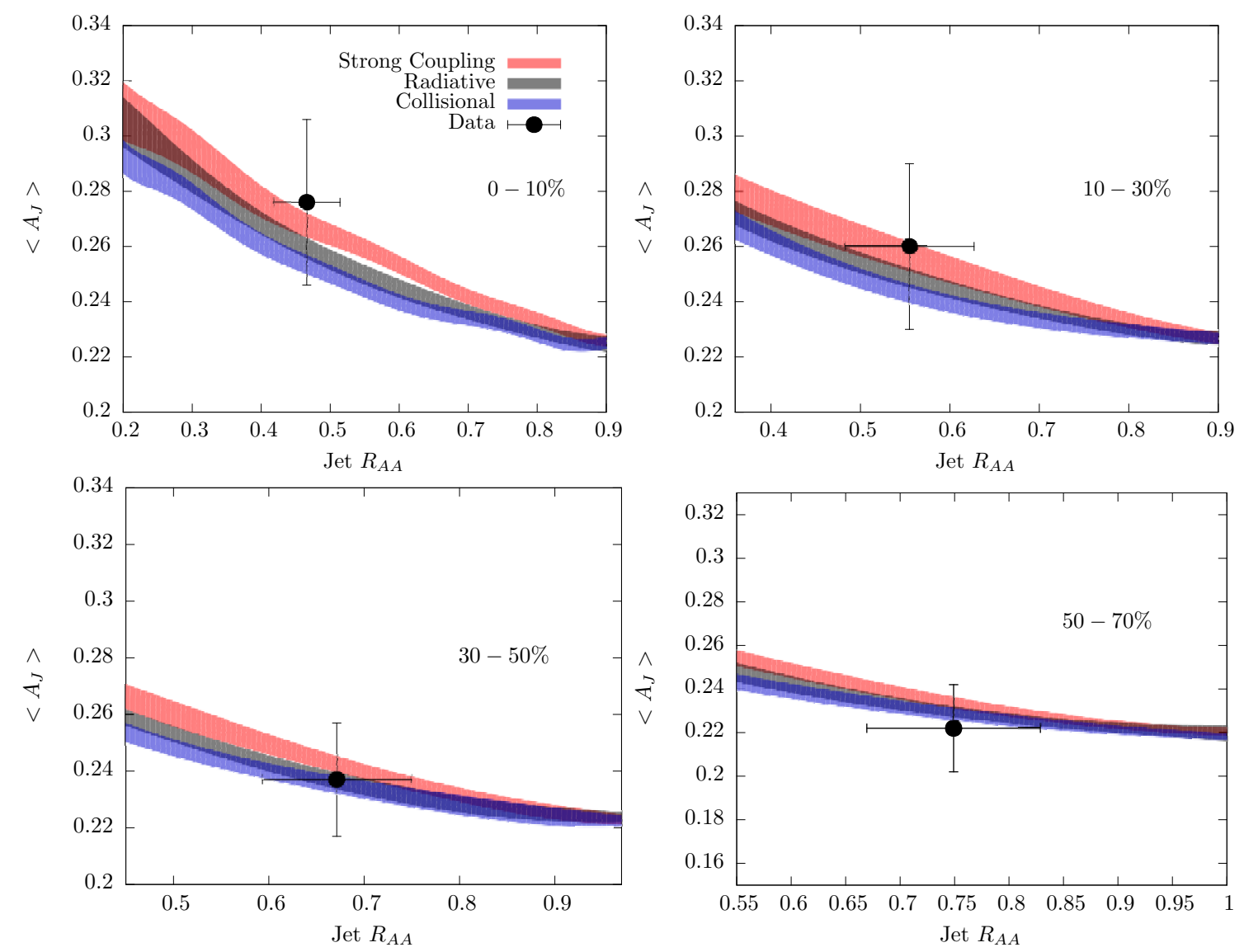

Figure 7. Mean di-jet imbalance as a function of jet suppression for three different models of jet energy loss. Data points are extracted from refs. [80] and [84].

As before, before over-interpreting the good agreement between the strongly coupled hybrid model prediction for the dijet asymmetry distribution and the data, in figure 6 we show the (smeared) results for the dijet asymmetry distribution in events with two different ranges of centrality if we use the strongly coupled (red), radiative (grey) and collisional (blue) models for the rate of energy loss $d E / d x$. As in the case of the jet suppression $R_{\mathrm{AA}}$, our results for the $A_{J}$ distribution is only weakly dependent on our choice of the underlying model. Even though the three different models have quite different path-length dependence for $d E / d x$, all three models lead to similar dijet asymmetries. Although it is a small effect, we do notice here that the strongly coupled model yields a slightly larger dijet imbalance in the most central collisions and that this means it is in somewhat better agreement with the data than the other two models. Nevertheless, the larger message of figure 6 is the approximate agreement between the predictions of three models with energy loss rates that feature very different path-length dependence, indicating that the these types of jet observables have only limited sensitivity to the shape of the underlying medium, as observed previously in ref. [86]. 


\subsection{Jet $R_{\mathrm{AA}}$ and mean dijet asymmetry}

In order to get a better sense of the (in)sensitivity of the two classes of jet observables that we have investigated so far to the mechanism by which the jet loses energy, and in particular in order to look separately at the consequences of theoretical and experimental uncertainties, in figure 7 we perform a parameter scan of the three models. For each model, we perform a series of simulations with varying values of the free parameter $\left(\kappa_{\mathrm{sc}}, \kappa_{\mathrm{rad}}\right.$ or $\left.\kappa_{\text {coll }}\right)$ in the expression for the energy loss rate $d E / d x$ (namely eq. (3.1), (3.6) or (3.9)) and compute both the mean asymmetry $\left\langle A_{\mathrm{J}}\right\rangle$ (for all dijet pairs with $p_{\mathrm{T} 1}>120 \mathrm{GeV}$ and $p_{\mathrm{T} 2}>30 \mathrm{GeV}$ ) and the jet suppression factor $R_{\mathrm{AA}}$ (for jets with $100 \mathrm{GeV}<p_{\mathrm{T}}<110 \mathrm{GeV}$ ) in a given centrality bin. Each such scan over the value of the free parameter in one of the models yields a curve in the $\left(\left\langle A_{\mathrm{J}}\right\rangle, R_{\mathrm{AA}}\right)$ plane. For each model, we obtain a band in figure 7 that gives a sense of the theoretical uncertainty within the given model by varying the critical temperature $T_{c}$ arising in the hydrodynamic solution, as described in section 4 . The different path length dependence of the three energy loss mechanisms is, in principle, reflected in the different shapes of the bands displayed in figure 7 . These differ the most in the most central bin where, despite the width introduced by the theoretical uncertainty, the different behavior in the different models is distinguishable. However, the differences between the models are small compared to the present experimental uncertainties. Consistent with what we have seen in figure 6 , for $R_{\mathrm{AA}}$ values close to the experimental ones the mean asymmetry of the three models is similar, but with the strongly coupled model yielding slightly bigger asymmetries. For comparison, we have also plotted the corresponding experimental data points which we extracted ${ }^{6}$ from refs. [80] and [84].

The large systematic uncertainty in the determination of the mean ratio $\left\langle p_{\mathrm{T} 2} / p_{\mathrm{T} 1}\right\rangle$ that determines $\left\langle A_{J}\right\rangle$ is responsible for the largest part of the experimental error bars displayed in figure 7 . These large error bars, combined with the smallness of the separation between the bands corresponding to the different models, makes it impossible to use this analysis to favor any of the models with any confidence. However, the range of model parameters which can simultaneously accommodate the measured values of the jet suppression and the dijet asymmetry is larger for the strongly coupled model. This corresponds to the slightly better fit to the dijet asymmetry data provided by the strongly coupled model in figure 5 . Perhaps the data therefore favor the strongly coupled model very slightly. At present, however, these data do not really discriminate among the models that we have explored, given the current error bars and given the similarity between the predictions of these three (very different) energy loss models for the $R_{\mathrm{AA}}$ and $A_{J}$ observables. Although one could investigate whether the separation between the bands in figure 7 can be increased by using different ranges of $p_{\mathrm{T}}$ in the evaluation of $R_{\mathrm{AA}}$ or $\left\langle A_{J}\right\rangle$, we do not anticipate reaching different conclusions until a time when the uncertainties in jet measurements at the LHC have been substantially reduced.

\footnotetext{
${ }^{6}$ Since CMS uses different centrality bins for its $\left\langle A_{J}\right\rangle$ and $R_{\mathrm{AA}}$ measurements, we combined the experimental values of $R_{\mathrm{AA}}$ from CMS' $0-5 \%$ and $5-10 \%$ centrality bins, and their measurements of $\left\langle A_{J}\right\rangle$ for their $10-20 \%$ and $20-30 \%$ bins. In each such combination, we weight the value of the observable in each of the smaller centrality bins that we are combining by the ratio of the number of jet events in that bin to the total number of jet events in the larger combined bin. We extract these ratios from the forward calorimeter energy deposition distributions in jet triggered $\mathrm{Pb}-\mathrm{Pb}$ events shown in ref. [84].
} 

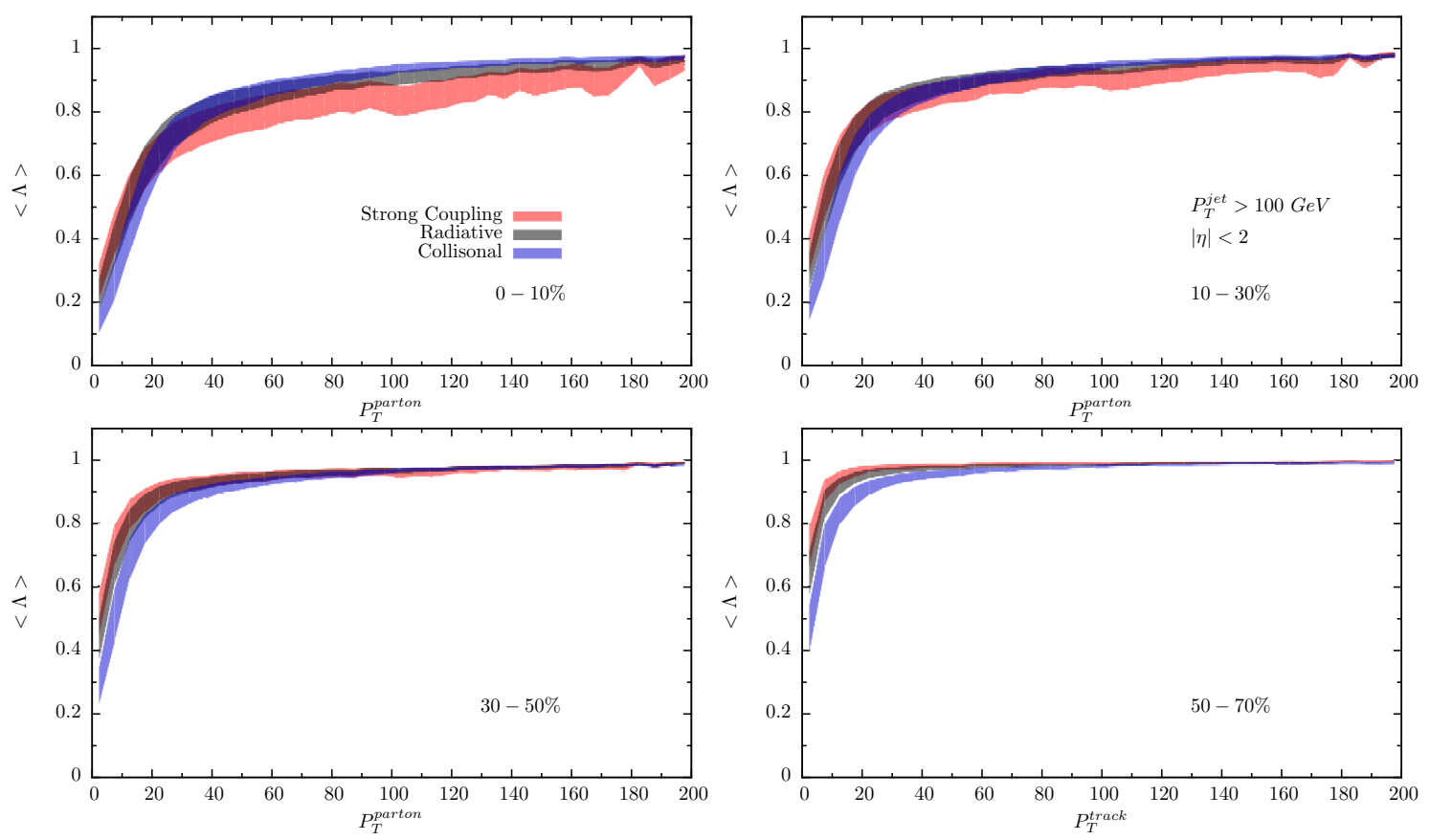

Figure 8. Mean value of the ratio $\Lambda$ between the transverse momentum $p_{T}^{\text {parton }}$ of a parton in the reconstructed quenched jet to the transverse momentum that that parton would have had in the absence of any medium. We plot $\langle\Lambda\rangle$ as a function of $p_{T}^{\text {parton }}$ for jets with $p_{\mathrm{T}}>100 \mathrm{GeV}$ in $\mathrm{Pb}-\mathrm{Pb}$ collisions at the LHC in four centrality bins. $\Lambda$ is not experimentally measurable; investigating it nevertheless allows us to discern the effects of the differences between models.

\subsection{Energy lost by individual partons within a jet}

The insensitivity of the inclusive jet observables that we have looked at so far leads us to consider more differential quantities. We start by studying the distribution of the energy lost by the individual partons within a reconstructed jet, although this distribution itself is not an experimental observable. In figure 8 we show the mean value of the ratio $\Lambda$ of the transverse momentum of a parton after it has been quenched by propagating through the plasma, $p_{T}^{\text {parton }}$, to the transverse momentum that that parton would have had in the vacuum PYTHIA jet absent any quenching. We plot $\langle\Lambda\rangle$ as a function of the $p_{\mathrm{T}}$ of the parton, averaged over all the partons in the jets with total $p_{\mathrm{T}}>100 \mathrm{GeV}$ in four different centrality bins. The average takes into account the fluctuation in $\Lambda$ induced both by variations in the path length through the medium traversed by different jets as well as by the different pattern and times of branching that can result in a parton in the final state jet with a given parton momentum. Although $\Lambda$ is not measurable, since knowing $\Lambda$ requires knowledge of the momentum that a parton would have had if there had been no medium present, it provides us with information as to where differences among different models arise.

For all three models, the rough features of the distributions in figure 8 are qualitatively the same. At high momentum, all the models feature a reasonably momentum independent $\langle\Lambda\rangle$ which saturates at the highest momenta at roughly comparable values in all the models. This is a consequence of our fitting procedure: we have fixed the one free parameter in each 
of the models so as to correctly describe $R_{\mathrm{AA}}$ in a certain $p_{\mathrm{T}}$ and centrality bin; because of the steeply falling jet spectrum, $R_{\mathrm{AA}}$ is only sensitive to whether jets that start out with a given $p_{\mathrm{T}}$ lose even a small amount of energy, not to how much energy these jets lose on average and not to the energy lost by jets that begin with higher $p_{\mathrm{T}}$; this in turn means that $R_{\mathrm{AA}}$ is most sensitive to the energy loss experienced by the hardest partons in a jet; so, by fitting the parameter in each model to $R_{\mathrm{AA}}$ we end up with the models having quite similar $\langle\Lambda\rangle$ at high parton momentum. Turning now to low parton momenta, all three models also efficiently quench soft partons. For the collisional and radiative models, this is a consequence of the fact that the energy loss rate $d E / d x$ in (3.6) and (3.9) is independent of the energy of the parton $E$. This means that when we fix the parameter $\kappa_{\text {rad }}$ or $\kappa_{\text {coll }}$ by fitting to $R_{\mathrm{AA}}$, ensuring some nonzero fractional energy loss for the highest momentum partons, we end up with a larger fractional energy loss for the lower momentum partons. For the strongly coupled model, the quenching of soft partons is enhanced by the Bragg-like behavior of the energy loss, with $d E / d x$ in (3.1) rising rapidly as $x$ approaches $x_{\text {stop }}$ and the parton becomes soft.

While the basic qualitative features of the $\langle\Lambda\rangle$ versus parton $p_{\mathrm{T}}$ curves plotted in figure 8 are similar for all three models, the quantitative shapes of the curves are different for the different models. The strongly coupled energy loss model yields a flatter curve than do the other models, with a lower asymptotic value at high momentum and softer turn over for the most quenched partons than in the other two models. The collisional model, in which $d E / d x$ has no path length dependence, has the steepest behavior in figure 8 . This correlation between the path length dependence of the energy loss model and the behavior of the $\langle\Lambda\rangle$ curves in figure 8 is easy to understand. Softer partons are in general created later and so travel less distance in the plasma, meaning that if the rate of energy loss $d E / d x$ increases with distance traveled, as in the case of weakly coupled radiative energy loss (3.6) or the strongly coupled energy loss (3.1), the fractional energy lost by the soft partons is less than in the case of collisional energy loss, where $d E / d x$ is independent of $x$. So, the more pronounced the $x$-dependence of $d E / d x$ the flatter the $\langle\Lambda\rangle$ vs. $p_{\mathrm{T}}{ }^{\text {parton }}$ curve in figure 8 should be, as indeed is seen in the figure. This more pronounced path length dependence is also responsible for the larger width of the theoretical uncertainties, since the relative enhancement of late time quenching makes the model more sensitive to the temperature at which quenching is turned off.

\subsection{Fragmentation function ratio}

We have seen that inclusive jet observables like the jet suppression factor $R_{\mathrm{AA}}$ and the dijet asymmetry $A_{J}$ are not particularly sensitive to the differences between the three energy loss models that we are investigating. We have also seen, however, that if we look at differences between the behavior of partons within the jets with different $p_{\mathrm{T}}$ we can find consequences of the different energy loss models. This motivates us to investigate jet fragmentation functions. From our model, we can compute partonic fragmentation functions, which are probability distributions for the fraction of the total jet momentum that is carried by an individual parton in the final state jet. In figure 9 , we show the ratio of the partonic fragmentation function for the quenched jets in our hybrid strongly coupled model with $d E / d x$ as in (3.1), as well as for the analogous weakly coupled radiative and collisional 

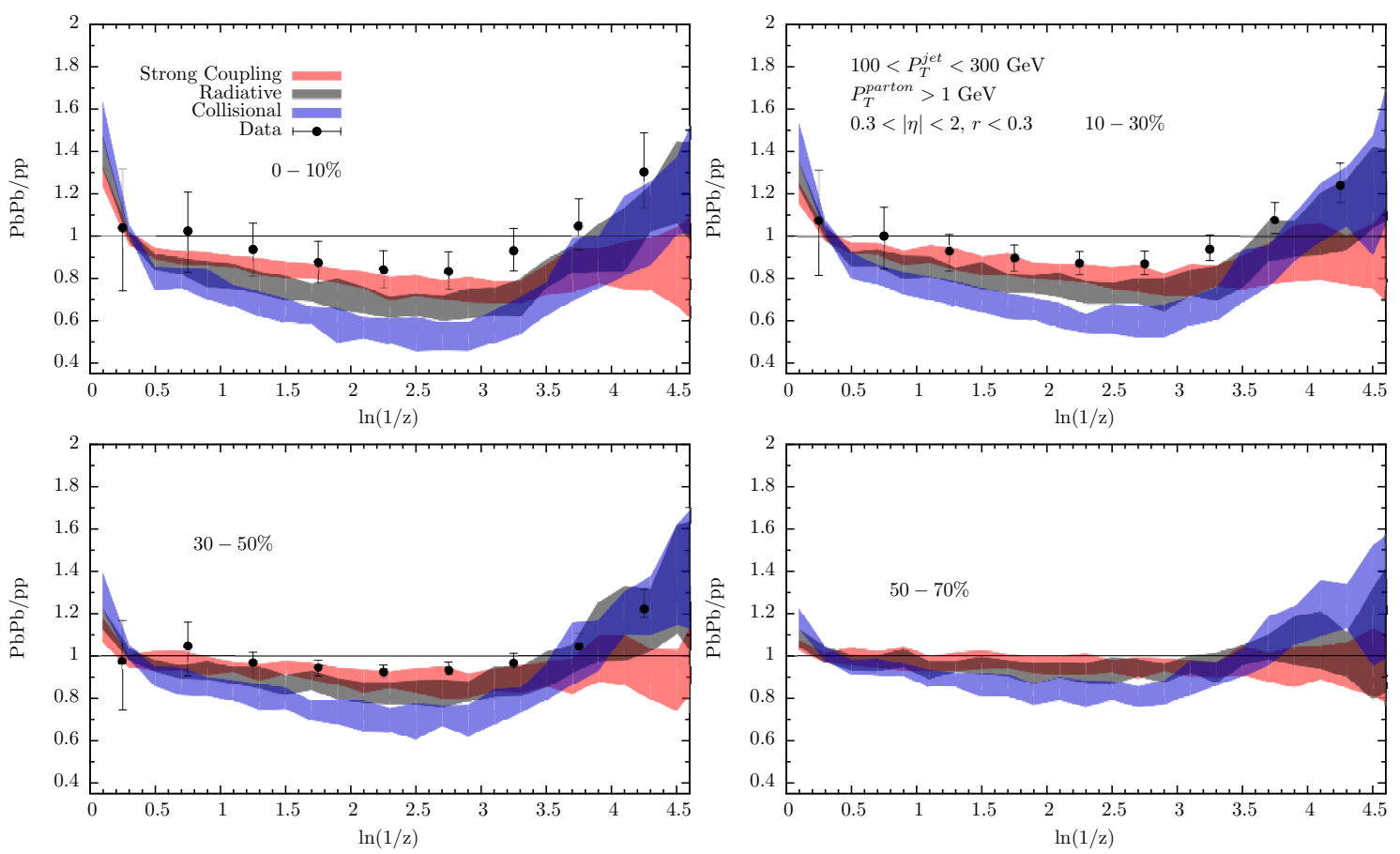

Figure 9. Ratio of the partonic level fragmentation function for our quenched jets to that for proton-proton jets in the same reconstructed jet $p_{T}$ interval $100<p_{T}<300 \mathrm{GeV}$, for jets with $0.3<|\eta|<2$ and for different centrality bins. The data points are the analogous experimentally measured fragmentation function ratio from ref. [88], for hadrons rather than partons.

energy loss models with $d E / d x$ as in (3.6) and (3.9), to the fragmentation function for a PYTHIA jet in vacuum with the reconstructed jet energy in the same interval as for the quenched jets. This ratio is analogous to the fragmentation function ratio measured in $\mathrm{Pb}-\mathrm{Pb}$ collisions at the LHC by both ATLAS and CMS [87-89], but of course they measure the fraction of the total jet momentum that is carried by individual hadrons whereas our calculation is performed at the parton level. As in the experimental analysis in ref. [87, 88], we determine the jet axis and momentum using the anti- $k_{t}$ algorithm with $R=0.3$ and we then search for all the partons in a cone with radius $r \equiv \sqrt{(\Delta \phi)^{2}+(\Delta \eta)^{2}}<0.3$ centered on the jet axis and use the projection of the parton momentum onto the jet axis to define $z=$ $p_{\|}^{\text {parton }} / p^{\text {jet }}$. Since we have stopped the DGLAP evolution in PYTHIA for each parton that reaches a minimum virtuality $Q_{0}=1 \mathrm{GeV}$, we stop our computation of the fragmentation function ratio in figure 9 at $\ln (1 / z)=\ln (100)=4.6$. This also reduces our sensitivity to the effects of hadronization on the fragmentation function, which we are leaving out of our partonic calculation. For $z$ values smaller than our cut, the effects of hadronization become more important, since the dynamics of hadronization can soften particles below $1 \mathrm{GeV}$.

The overall message from figure 9 is that the fragmentation function of a quenched jet is very similar to that of a vacuum jet with the same energy as the quenched jet. This was first pointed out in ref. [87] and remains the case in the data from ref. [88] that we have shown in the figure, and it is also the case for our hybrid strongly coupled model. The collisional model that we have set up as a foil in this paper does not share this feature. 
The fragmentation function ratio predicted by the collisional model lies below the data over several decades of $1 / z$, meaning that this model can be ruled out by the comparison of its fragmentation function to the data. The fragmentation function ratio predicted by our hybrid strongly coupled model does best, comparing very well with the data in figure 9 . The weakly coupled radiative model fares in between, predicting a fragmentation function ratio that is in some tension with the data, particularly in mid-centrality collisions. It should be noted that since in the case of radiative energy loss the 'lost' energy is expected to include some moderate- $p_{\mathrm{T}}$ particles that initially stay within the jet cone, the assumption that we are making in our implementation of this model that all of the 'lost' energy becomes soft particles moving in directions that are uncorrelated with the jet direction may be suspect here. If so, our calculation of the fragmentation function in our implementation of radiative energy loss is incomplete.

Note that in comparing our model calculations of the fragmentation function ratio to data in figure 9 we are ignoring the softest part of the fragmentation function ratio shown in the figure. We do so for two reasons. First, although we have ended our partonic calculation at $Q_{0}=1 \mathrm{GeV}$ and cut the figure of at $z=1 / 100$ precisely to reduce this problem, comparison of our partonic fragmentation functions to the data on hadronic fragmentation functions may not be appropriate at the smallest $z$ 's we have plotted, given that hadronization tends to soften softer partons. Second, the low $p_{\mathrm{T}}$ particles that populate the smallest $z$ region that we have plotted in figure 9 have momenta that are small enough that many of them could certainly come from the thermal distribution of particles formed as the quark-gluon plasma cools and hadronizes. The background subtraction procedure used in the analyses of experimental data will subtract such particles, on average, if they are uncorrelated with the jet direction. This subtraction may not be perfect, however, either because of fluctuations in the bulk droplet of plasma or because some of the energy lost by the jet, which we are assuming ends up as a little hotter or a little extra plasma, may also manifest itself in collective motion of the plasma, meaning that although the 'lost' energy becomes soft particles these soft particles might not be completely uncorrelated with the jet direction. For both these reasons, the subtraction of whatever fraction of the 'lost' energy ends up in the jet cone may not be complete. We have checked that adding only one soft particle per jet can result in a substantial upturn in the fragmentation function ratio at $\ln (1 / z) \gtrsim 4$, and for this reason we will not compare to the data in this regime.

The main features of the fragmentation functions displayed in figure 9 can be understood from the distribution of quenching factors $\langle\Lambda\rangle$ shown in figure 8. At $z \rightarrow 1$ the quenched (partonic) fragmentation function is close to the vacuum one, with only a small enhancement observed. This enhancement is a consequence of the depletion of soft fragments observed in all the models, which tends to make the in-medium fragmentation functions harder than in vacuum. While this enhancement is present in all three models, it is smallest in the strongly coupled model, since the quenching factor $\langle\Lambda\rangle$ is least dependent on the $p_{\mathrm{T}}{ }^{\text {parton }}$ in this model, see figure 9 . At intermediate $z$, all the in-medium fragmentation functions are depleted relative to the vacuum fragmentation functions. The $z$-values where such depletion starts are correlated with the transverse momentum below which the quenching factor $\langle\Lambda\rangle$ drops in figure 8. In the collisional and radiative models, 
this occurs at a higher momentum and, as a consequence, the intermediate- $z$ depletion in the fragmentation function ratio is larger in these two models than for the strongly coupled model. The distinctions between the fragmentation function ratios of the three models at the lowest $z$ 's plotted in figure 9 can also be understood in terms of features of figure 8 , but we have already explained why we will not focus on this region.

We have observed that the collisional model leads to a much stronger depletion of the quenched fragmentation functions relative to what is measured in data, over several decades of $z$. This is a direct consequence of the lack of path-length dependence in $d E / d x$ in this model, meaning that our conclusion that this model is disfavored seems robust. The radiative model seems to be marginally in agreement with the data. Remarkably, the weaker modification of the in-medium fragmentation function within the strongly coupled hybrid model achieves the best qualitative agreement with the fragmentation function ratio in the experimental data. A more quantitative, and more definitive, statement along these lines would require including hadronization in our strongly coupled hybrid model, would require investigating where the energy 'lost' by the jet ends up rather than just assuming that it becomes soft particles uncorrelated with the jet direction, and would require including the soft particles corresponding to the plasma itself in our model and subtracting them during jet reconstruction as in the analyses of experimental data. We leave all these investigations to future work.

\section{Conclusions, discussion and a look ahead}

\subsection{Conclusions}

We have seen in section 5 that our hybrid approach, with perturbative QCD (via PYTHIA) describing the parton splitting that occurs within a jet while at the same time each parton in the jet loses energy according to the expression (3.1) for $d E / d x$ for a light quark traveling through strongly coupled plasma, derived via a holographic calculation in ref. [45], is very successful in describing the available jet data at the LHC. After fixing the one free parameter in the model, defined in (3.2), using the measured value of the suppression factor $R_{\mathrm{AA}}$ for jets in one $p_{\mathrm{T}}$-bin in the most central $\mathrm{Pb}-\mathrm{Pb}$ collisions at the LHC, we obtain a completely satisfactory description of the dependence of the jet $R_{\mathrm{AA}}$ on both $p_{\mathrm{T}}$ and centrality as well as of the dijet asymmetry $A_{J}$, including its centrality dependence. In addition, we make predictions for the jet $R_{\mathrm{AA}}$ at RHIC. We also find that the (small) deviations between the fragmentation functions of quenched jets measured in heavy ion collisions at the LHC and those of vacuum jets with the same energy as the quenched jets compare very well with the corresponding fragmentation function ratios described by our hybrid model.

The above successes are important, but they should not be over-interpreted. The current uncertainties in the measurements of jet $R_{\mathrm{AA}}$ translate into a significant dispersion in our theoretical computations, reflected in the width of all the colored bands in our plots in section 5. And, partly as a consequence of these uncertainties and partly as a consequence of the insensitivity of inclusive jet observables to the mechanism by which energy is lost, we have found that present measurements of the jet suppression factor $R_{\mathrm{AA}}$ and the dijet asymmetry $A_{J}$ are described almost as well if we use the models for $d E / d x$ motivated by weakly 


\begin{tabular}{|c|c|c|c|}
\hline & Strong Coupling & Radiative & Collisional \\
\hline Parameter & $0.26<\kappa_{\mathrm{sc}}<0.35$ & $0.81<\kappa_{\mathrm{rad}}<1.60$ & $2.5<\kappa_{\text {coll }}<4.2$ \\
\hline
\end{tabular}

Table 1. Values of the fit parameters needed in the specification of $d E / d x$ in our three different energy loss models, in each case as extracted by comparing model predictions for $R_{\mathrm{AA}}$ for jets with $100 \mathrm{GeV}<p_{\mathrm{T}}<110 \mathrm{GeV}$ in central $\mathrm{Pb}-\mathrm{Pb}$ collisions at the LHC to experimental data.

coupled radiative or collisional energy loss that we have described in section 3 . The comparisons between the partonic fragmentation function ratios that we can compute in our models and the fragmentation functions measured at the LHC that we have made in section 5.5 do favor the hybrid strongly coupled approach over the model with collisional energy loss and, to some degree, over the model with radiative energy loss. However, this is a comparison between a partonic calculation and a hadronic measurement, so perhaps we should not take the fact that the data favors the strongly coupled energy loss rate as definitive.

The success (or partial success in the collisional case) of all these energy loss mechanisms, which arise from very different pictures of the underlying dynamics, crucially depends on the freedom to choose the overall strength of energy loss by fitting one model parameter to data. It therefore becomes important to confront the parameters extracted from data to expectations from theoretical calculations. We shall do this in section 6.2. We close in sections 6.3 and 6.4 with a look ahead in two senses, first with various ways that our study could be improved and, second, with a suggestion for an additional, more incisive, observable.

\subsection{Significance of the extracted parameters}

The three models for $d E / d x$ that we have tested in this paper each include one free parameter that we have fitted to experimental measurements of $R_{\mathrm{AA}}$ for jets with $100 \mathrm{GeV}<$ $p_{\mathrm{T}}<110 \mathrm{GeV}$ in central $\mathrm{Pb}-\mathrm{Pb}$ collisions at the LHC. We have collected the values of these parameters obtained via fitting to this data in table 1. See eqs. (3.2), (3.6) and (3.9) for the definitions of the parameters.

The values of $\kappa_{\text {rad }}$ and $\kappa_{\text {coll }}$ in the weakly coupled radiative and collisional models for $d E / d x$ obtained via our fit to data should be compared to expectations based upon perturbative calculations given in eqs. (3.7) and (3.8) and in eqs. (3.10) and (3.11). We see that our fit to data corresponds to a value of the strong coupling constant $\alpha_{s}$ that is smaller (larger) than the range $0.2<\alpha_{s}<0.3$ that we used in making the estimate (3.8) for $\kappa_{\text {rad }}$ (the estimate (3.11) for $\kappa_{\text {coll }}$.) In the case of radiative energy loss, as we discussed in section 3 it may be that we are underestimating $\kappa_{\text {rad }}$ because we are neglecting the fact that much of the 'lost' energy is initially radiated in the form of gluons moving in the same direction as the jet, meaning that some of this radiated energy may remain correlated with the jet direction. If this is so, by neglecting this we would be overestimating the energy loss at a given $\kappa_{\text {rad }}$ and hence our fit would be underestimating $\kappa_{\text {rad }}$.

As we have discussed in section 3 and as is manifest in eqs. (3.7) and (3.10), because of rare radiative or collisional processes in which a large momentum is transferred the perturbative evaluation of $\kappa_{\text {rad }}$ or $\kappa_{\text {coll }}$ leads to logarithms of ratios of scales, $B_{\text {rad }}$ and 
$B_{\text {coll }}$, which may depend on the kinematics of the colliding objects and whose evaluation is beyond the accuracy of current theoretical calculations. Since the precise expressions for both $B_{\text {rad }}$ and $B_{\text {coll }}$ are unknown, it is best to think of our fits to data as constraining the product of the appropriate power of $\alpha_{s}$ times the appropriate large logarithm, as in the middle expressions in eqs. (3.8) and (3.11). Our fits yield relatively large values for this product, both in the case of radiative energy loss and in the case of collisional energy loss. If the logarithmic corrections were small, as would be required for the simple perturbative expansion to be accurate, our analysis would yield such large values of $\alpha_{s}$ that perturbation theory would clearly be invalid. Or, if small values of $\alpha_{s}$ are chosen, as in the last expressions in eqs. (3.8) and (3.11), then the logarithms become large which again invalidates the simple perturbative expansion, in this case pointing towards the need for a resummation as discussed in refs. [53-55]. Note also that despite our simplified approach to energy loss, our results are compatible with those of more sophisticated approaches, such as those described in ref. [52], when the large logarithms are evaluated as prescribed in those works. The bottom line for the two weakly coupled models that we have introduced as benchmarks is that within our model context they can describe LHC data on jet $R_{\mathrm{AA}}$ and the dijet asymmetry $A_{J}$ if we choose values of the single parameter in each model that correspond to values of $\alpha_{s}$ that are large enough to make the reliability of a perturbative calculation questionable. At the same time, as we saw in section 5.5 the collisional model cannot reproduce LHC data on the fragmentation function ratio and the radiative model is in some tension with this data, at best in marginal agreement with it.

We now turn to the strongly coupled model. The comparison of the value of $\kappa_{\mathrm{sc}}$ that we have obtained via fitting our results to jet observables measured in heavy ion collisions at the LHC to the value obtained in theoretical calculations performed holographically, i.e. via gauge/gravity duality, is of necessity uncertain. The holographic calculations that we have employed were done in large- $N_{c}$, strongly coupled $\mathcal{N}=4$ SYM theory, not in QCD. There are by now large classes of theories with known gravitational duals, but the gravitational dual of QCD itself (if one exists) is not known. Present holographic calculations are therefore best used to gain qualitative insights, like for example the form of $d E / d x$ in (3.1) and the parametric dependence of $x_{\text {stop }}$ in (3.2). But there is no one right answer for how to compare a numerical value of $\kappa_{\mathrm{sc}}$ extracted via comparison to experimental measurements - of course in QCD - to a numerical value of $\kappa_{\text {sc }}$ computed in $\mathcal{N}=4$ SYM theory. That said, it is a generic expectation that the stopping distance $x_{\text {stop }}$ will be longer, meaning that $\kappa_{\mathrm{sc}}$ will be smaller, in strongly coupled QCD plasma than in strongly coupled $\mathcal{N}=4$ SYM plasma with the same temperature because QCD has fewer degrees of freedom than $\mathcal{N}=4 \mathrm{SYM}$ theory by a factor $\approx 0.4$. There are various prescriptions in the literature for how this reduction in the energy density of the plasma at a given temperature may affect holographic calculations of various quantities, but this has not been investigated for the stopping distance of a light quark. And, of course, the QCD plasma differs from that in $\mathcal{N}=4$ SYM theory in other ways also.

The comparison of the value of $\kappa_{\mathrm{sc}}$ that we have extracted via comparison with data to theoretical expectations originating in holographic calculations is further complicated by the fact that, as we have discussed in section 3, theorists have developed several different 
ways of modeling jets in $\mathcal{N}=4 \mathrm{SYM}$ theory, given that jets are not actually produced in hard processes in this theory. Different values of $\kappa_{\mathrm{sc}}$ are obtained in $\mathcal{N}=4$ SYM theory depending on whether a jet is modeled as a single string moving through the plasma, in which case $\kappa_{\mathrm{sc}}{ }^{\mathcal{N}=4}=1.05 \lambda^{1 / 6}$ [22], or via analyzing the decay of a virtual external $\mathrm{U}(1)$ field into $\mathcal{N}=4 \mathrm{SYM}$ matter with initial virtuality $q$ and initial position in the holographic direction $D / q$ with $D$ an unknown factor that is of order unity, in which case $\kappa_{\mathrm{sc}}{ }^{\mathcal{N}=4}=1.24 D^{1 / 3}[24]$. Although these two estimates of $\kappa_{\mathrm{sc}}$ differ parametrically, the first being of order $\lambda^{1 / 6}$ while the second is of order unity, their numerical values are similar. If we set $N_{c}=3$, the 't Hooft coupling is $\lambda \equiv g^{2} N_{c}=12 \pi \alpha_{s}$ meaning that if we choose $0.2<\alpha_{s}<0.3$ this corresponds to $7.5<\lambda<11.3$ or $1.4<\lambda^{1 / 6}<1.5$. So, combining the two estimates, we learn that if we apply an $\mathcal{N}=4$ SYM theory calculation done with $N_{c} \rightarrow \infty$ and $\lambda \rightarrow \infty$ to $\mathcal{N}=4$ SYM theory with $N_{c}=3$ and $7.5<\lambda<11.3$ we conclude

that $1.2 \lesssim \kappa_{\mathrm{Sc}} \mathcal{N}=4 \lesssim 1.6$, with the lower end of the range uncertain by a factor that is of order unity. From this we conclude that the value of $\kappa_{\mathrm{sc}}$ that we have extracted by comparing our results to experimental data on $R_{\mathrm{AA}}$ for jets in the QCD plasma produced in LHC collisions is smaller than that in $\mathcal{N}=4$ SYM theory by a factor of about $1 / 3$ to $1 / 4$, meaning that $x_{\text {stop }}$ is longer in the QCD plasma produced in a heavy ion collision than in the $\mathcal{N}=4 \mathrm{SYM}$ plasma by a factor of about 3 to 4 .

We conclude that the hybrid strongly coupled approach to jet quenching that we have developed is in good agreement with all the various measured jet observables to which we have compared it in section 5 when we take all the parametric dependence of $d E / d x$ and $x_{\text {stop }}$ from the expressions (3.1) and (3.2) derived for the $\mathcal{N}=4$ SYM plasma, and set the numerical value of $x_{\text {stop }}$ in the QCD plasma longer than that in the $\mathcal{N}=4 \mathrm{SYM}$ plasma as expected, longer by a factor of 3 to 4 .

\subsection{Opportunities for improvements to our implementation}

Although we have found that the inclusive jet observables $R_{\mathrm{AA}}$ and $A_{J}$ have limited discriminating power in differentiating between different energy loss mechanisms, the success of the hybrid strongly coupled model that we have developed in describing these data is encouraging. The comparative success of the hybrid strongly coupled model relative to the radiative model and, in particular, relative to the collisional model in describing the data on fragmentation function ratios provides further encouragement. What we have done is, however, only an initial exploratory study. We are much more confident in the value of our hybrid approach than in the specifics of the model implementation that we have pursued in detail because we have made many simplifying assumptions in implementing our hybrid approach. Here we summarize some of the main simplifications, all of which represent opportunities for future improvements. Such improvements are well motivated indeed, given the increase in the quantity and quality of data on jet observables at both the LHC and RHIC anticipated in the near future.

Some of the improvements that should be investigated come from the phenomenological aspects of our model. For example, our study should be repeated using solutions to three-dimensional viscous hydrodynamics rather than the boost-invariant solution to ideal hydrodynamics that we have employed. And, the effects of adding hadronization to 
the model should be studied, as although this would open up new uncertainties it would also open up the possibility of comparing to new observables. As we have discussed in section 5.5, it would be of considerable interest to try to follow the energy lost by the quenched jet and to investigate the degree to which the fraction of the 'lost' energy that happens to become soft particles within the jet cone is or is not subtracted during the jet reconstruction procedure used in the analysis of experimental data.

There are other improvements that should be investigated that reside within the holographic calculations that yield results like (3.1) that we have employed. This list is fairly standard, applying just as much here as in the many other contexts in which holographic calculations have been employed to gain qualitative insights into strongly coupled gauge theory plasma and the dynamics of heavy ion collisions. For example, one can ask about finite $N_{c}$, finite $\lambda$, and nonzero $N_{f} / N_{c}$ corrections to (3.1), or about how this result changes in a strongly coupled theory that is not conformal.

The opportunities for improvement that are more unique to the approach that we have introduced in this paper reside in the hybridization of weakly coupled and strongly coupled dynamics that is at the core of our approach. To these we now turn.

A simple kinematic effect that we have neglected is the reduction in the phase space for the fragmentation of a parton in the PYTHIA shower as a consequence of the energy loss that we have added. Although we have assumed that the energy loss results from processes with small momentum transfer to or from the medium and therefore does not modify the probabilities for the hard splitting processes, in reality the reduction in the phase space for splitting will lead to some suppression in the rate of splitting. While this effect is small for the first energetic splittings, in the final stages of the shower it may be more significant. Given that $d E / d x$ in (3.1) increases with increasing $x$, making all the partons live a little longer will increase the effects of jet quenching if $\kappa_{\mathrm{sc}}$ is not modified which, in isolation, would reduce the fitted value of $\kappa_{\mathrm{sc}}$. At the same time, delaying splitting will reduce the number of partons in the shower which could reduce the effects of jet quenching for a give $\kappa_{\mathrm{sc}}$, resulting in an increase in the fitted value of $\kappa_{\mathrm{sc}}$.

There is a second effect that works in the opposite direction to the one above: as the partons in the shower interact via multiple soft interactions with the medium these interactions may induce additional splitting in the shower. Medium-induced splitting is of course at the core of the weakly coupled radiative energy loss mechanism. Adding this physics would push in the opposite direction to that above. It is hard to see, however, how this could be done without paying the price of introducing at least one further parameter that would have to be fit to data. One of the virtues of our present implementation is its minimalism. This improvement, and many of the other improvements that we enumerate here, would reduce the minimalism of the approach. As more data, more precise data and data on more observables, becomes available this may become a price worth paying.

A particularly important effect that we have not included in our computation is the kicks in transverse momentum (transverse to the initial jet direction) that the fragments in the shower will all pick up as they propagate through the medium, losing energy. For simplicity, we have assumed that all the in-medium partons maintain their direction of 
propagation. The inclusion of transverse momentum broadening would have little effect on $R_{\mathrm{AA}}$, which is dominated by the hardest fragments, and therefore would not have much effect on the extracted value of $\kappa_{\mathrm{sc}}$. However, as stressed in ref. [33], it would increase the dijet imbalance somewhat, since some of the soft fragments would get kicked out of the jet cone. We should mention, however, that this effect is unlikely to be pronounced because partons in the shower that become soft due to energy loss are very likely already being removed from the jet via the consequent large Bragg-like increase in $d E / d x$ in (3.1). Including transverse momentum broadening would make it possible to interpret other interesting observables. For example, in our present calculation our dijets are just as back-to-back as dijets in proton-proton collisions. This is consistent with present data on the distribution of the azimuthal angle separating jets in a dijet pair $[1,2,84]$ and the distribution of the azimuthal angle separating the photon and the jet in gamma-jet events [90]. However, at present it would not be sensible for us to compare our model to these data since there is no way within our model for these angular distributions to be different in $\mathrm{Pb}-\mathrm{Pb}$ collisions than in proton-proton collisions. After adding transverse momentum broadening to our model, we could then use the data that (at present) show no significant change in the distribution of the dijet or photon-jet azimuthal separation angle from proton-proton to $\mathrm{Pb}-\mathrm{Pb}$ collisions to constrain the new component of the model. We can further imagine using this data and a suitable variant of our hybrid model to separately constrain the probability that a hard parton is scattered by a large angle, thus looking for evidence of the presence of point-like quark and gluon quasiparticles [91]. So, incorporating transverse momentum broadening into our hybrid approach would result in a loss in minimalism and an increase in the number of parameters that would need to be fitted to data but it would mean that the model could be confronted with data on further observables, including the distributions we have just mentioned or, for example, various measures of jet shapes. We have made no attempt to analyze such observables in the present paper since medium-induced modification of jet shapes has to depend sensitively on transverse momentum broadening.

In this paper we have considered each of the three different expressions for the energy loss rate that we have investigated in isolation. Adding medium-induced splitting and transverse momentum broadening, which are both characteristic of radiative energy loss, to the hybrid strongly coupled model would be a step in the direction of combining the mechanisms that in this paper we have treated separately. After all, even when the typical interactions with the medium are soft and strongly coupled, with momentum transfers of order the temperature, the partons in the jet could have rare semi-hard interactions with constituents of the medium, inducing both gluon radiation and scattering of the parton by a substantial angle [91]. Looking for direct evidence of this in the data would be very interesting since at present there is no direct evidence for the presence of the weakly coupled point-like scatterers that, because QCD is asymptotically free, must be seen if the strongly coupled liquid quark-gluon plasma is probed at short enough distance scales. It is therefore worth modeling and, ideally, separating the effects of strongly coupled energy loss in conjunction with effects of occasional medium-induced gluon radiation and/or hard scattering. A further motivation for incorporating transverse momentum broadening is that even if the physics is entirely strongly coupled, multiple soft interactions add up to give nonzero transverse momentum broadening that can be substantial in magnitude [92, 93]. 
Another feature of the dynamics of energy loss that we have not implemented is the effects of finite resolution on the interaction between the shower and the medium. In a finite medium, the separation of the jet fragments in the transverse direction in position space as they propagate through the plasma must be finite. As has been explicitly shown for radiative processes [94], structures with a transverse size smaller than a given resolution scale must act coherently as seen by the medium. This reduces the effective number of propagating partons seen by the medium, and makes the 'effective partons' harder than anticipated. If $\kappa_{\mathrm{sc}}$ is left unchanged, these dynamics would tend to increase $R_{\mathrm{AA}}$, reduce the dijet asymmetry and make the fragmentation functions more similar to their vacuum counterparts. Of course, including these effects would result in a larger fitted value of $\kappa_{\mathrm{sc}}$. At present no implementation of the effects of finite transverse resolution is known at strong coupling, meaning that we have no evaluation of the appropriate resolution scale for a strongly coupled plasma and meaning that this investigation remains for the future.

Much remains to be done. It will be interesting to see how robust the conclusions of our study are as these further effects are included and as further observables become accessible within our hybrid approach.

\subsection{Distinctive species dependence and discriminating observables}

It is clearly important to find other less inclusive jet observables, in addition to the fragmentation function ratios that we have analyzed, that can be measured and that can further discriminate among different energy loss mechanisms. There is one salient, and quite possibly very significant, distinction between the models that we have introduced that we have not utilized at all: the dependence of the rate of energy loss $d E / d x$ on the color charge of the propagating hard parton. We have seen in section 3 that for both weakly coupled energy loss mechanisms, namely radiative energy loss as in (3.6) and collisional energy loss as in (3.9), the ratio of $d E / d x$ for quarks to that for gluons is $C_{F} / C_{A}=4 / 9$. In contrast, in the strongly coupled calculation the stopping distance (3.2) for quarks is longer than that for gluons only by a factor of $\left(C_{A} / C_{F}\right)^{1 / 3}$. This different color charge scaling means that even if parameters are chosen such that the overall magnitude of the energy loss is comparable in the different models, in the strongly coupled model the amount of energy lost by quarks and by gluons should be more similar to each other while in the weakly coupled models they should differ more. While the dependence of jet observables on this scaling is not straightforward to infer because a jet that is initiated by a quark contains many gluons in its fragments and vice versa, the difference among models as to how $d E / d x$ depends on $C_{F} / C_{A}$ will leave an imprint in the suppression pattern of jets initiated by quarks as compared to that of jets initiated by gluons.

In figure 10 we show the ratio of the number of quark-initiated jets to the number of gluon-initiated jets for jets with $100<p_{T}^{\text {jet }}<150 \mathrm{GeV}$ and pseudo rapidity $|\eta|<2$ as a function of centrality. The right-most point shows this ratio for vacuum jets. The centrality dependence of the ratio of the abundances of the two types of jets is a clear manifestation of the different rates of energy loss suffered by quark-initiated and gluon-initiated jets. For the hybrid strongly coupled model, whose $d E / d x$ depends most weakly on the parton's color charge, the ratio plotted in figure 10 is relatively close to its vacuum value for all the 


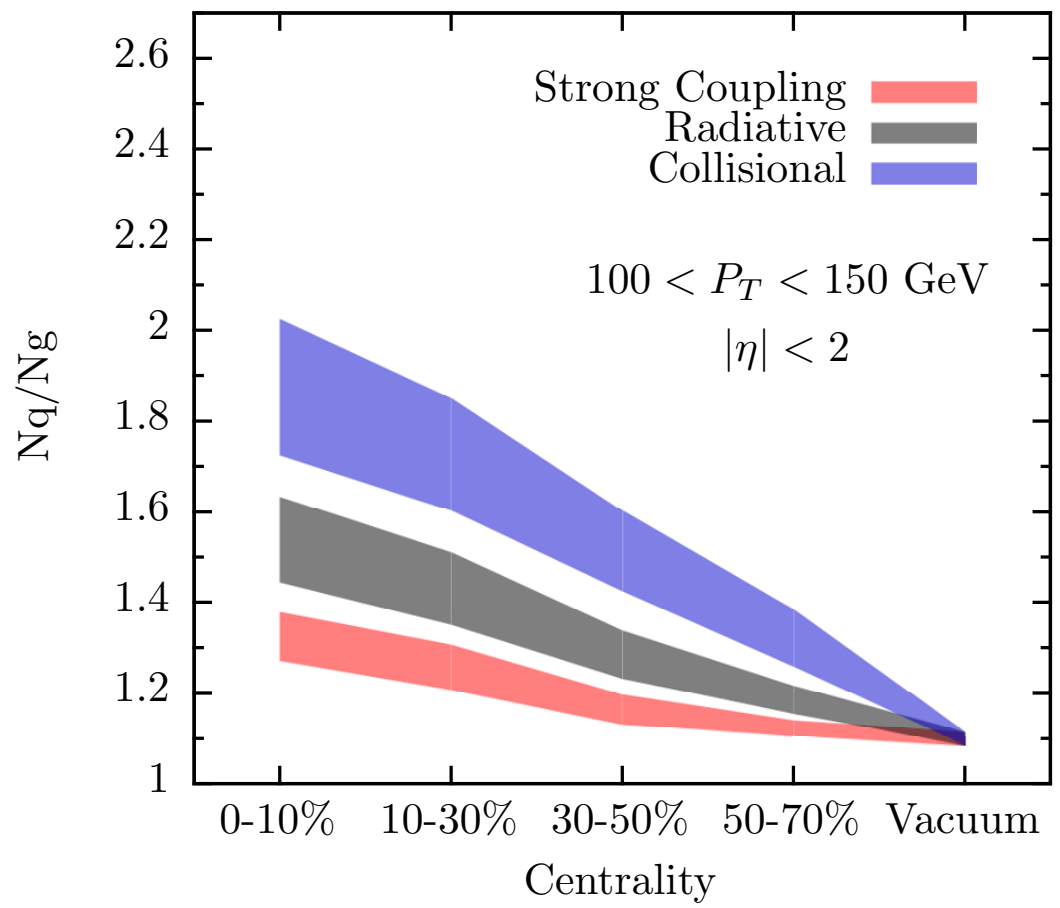

Figure 10. Predictions of our model, with the three different mechanisms for energy loss that we have investigated, for the ratio of the number of quark-initiated jets to the number of gluoninitiated jets as a function of centrality for jets whose transverse momentum, after quenching, lies in the range $100 \mathrm{GeV}<p_{\mathrm{T}}<150 \mathrm{GeV}$. The centrality dependence of this ratio is significantly smaller for our hybrid strongly coupled model than for either of the weakly coupled models.

centrality bins, reflecting the fact that the energy loss experienced by quarks and gluons is relatively similar in this model. In contrast, both the collisional and radiative models exhibit a much more pronounced centrality dependence in the ratio plotted in figure 10. In more central collisions in which jet quenching is more significant overall, the gluon-initiated jets suffer more energy loss than the quark-initiated jets because $d E / d x$ scales with $C_{A} / C_{F}$ and so the ratio of quark-initiated jets to gluon-initiated jets in a given $p_{\mathrm{T}}$-range must increase. The effect is greater in the collisional model than in the radiative model because, as we saw in figure 8 , in the collisional model the quenching of soft particles is particularly efficient and gluon jets tend to have a softer fragmentation pattern than quark jets.

From this study we conclude that, if it were possible for experimentalists to identify jets as quark-initiated or gluon-initiated, comparing the jet suppression factor $R_{\mathrm{AA}}$ for these two classes of jets would discriminate effectively between the three different models of energy loss that we have considered. Unfortunately, although there has been substantial recent progress toward separating quark-initiated jets and gluon-initiated jets in proton-proton collisions [95-100], doing so in heavy ion collisions is sufficiently challenging that it does not yet seem within reach. One exception is jets produced back-to-back with a hard photon [90], since these jets are predominantly quark-initiated jets. Extending the implementation of our hybrid approach to include gamma-jet events and using it to constrain the energy loss of quark-initiated jets relative to that of all jets, and hence to discriminate among models, 
will be of considerable interest. Another exception is b-tagged jets, a large fraction of which are b-quark-initiated jets. Data on the suppression factor $R_{\mathrm{AA}}$ for b-tagged jets with transverse momenta $p_{\mathrm{T}}$ between 80 and $250 \mathrm{GeV}$ [101] show no significant difference between their suppression and the suppression of inclusive jets in any of four centrality bins. Since at these very high values of $p_{\mathrm{T}}$ the mass of the b-quarks should have little effect, b-quarkinitiated jets in this regime are a good proxy for quark-initiated jets, meaning that the data [101] favor energy loss models in which $d E / d x$ for a parton depends only weakly on the color charge of that parton. Although at present the experimental error bars are large, these data already provide some further evidence in support of the hybrid strongly coupled model with $d E / d x$ as in (3.1). Reaching a firm conclusion also has to await further theoretical analysis of the energy loss of ultrarelativistic heavy quarks, and the jets initiated by them. Holographic calculations of the rate of energy loss of a heavy quark with mass $M$ moving slowly [18-20] (with a velocity such that its Lorentz boost $\gamma$ satisfies $\sqrt{\gamma}<M /(\sqrt{\lambda} T)[102-$ 104]) through strongly coupled plasma are well understood but the transition at larger $\gamma$ to the regime in which the heavy quark behaves like a light parton is not yet understood. Nevertheless, for b-quarks with $80 \mathrm{GeV}<p_{\mathrm{T}}<250 \mathrm{GeV}$ it should be reasonable to simply neglect the b-quark mass as we did above. Upon so doing, we reach the conclusion that b-quark jets, and hence quark jets, are quenched to the same degree as the mix of light-quark-initiated and gluon-initiated jets found in inclusive jets. This observation, together with the present data, favors the hybrid strongly coupled model for $d E / d x$, as we have discussed. This suggests that $R_{\mathrm{AA}}$ for b-jets, $A_{J}$ for dijets in which one or both of the jets are b-jets, and the b-jet fragmentation function ratio can, if measured in heavy ion collisions and analyzed via our hybrid approach, yield observables that discriminate effectively between energy loss models. With further development, and in particular with the investigation of these and other observables, the observation that at strong coupling energy loss depends more weakly on the type of parton (quark vs. gluon) may yield a robust signal for the strongly (or weakly) coupled nature of medium-induced jet quenching.

Much remains to be done and many further observables remain to be investigated. Further exploration of the hybrid approach that we have introduced in this paper and its implementation via the strongly coupled energy loss rate (3.1) and the stopping distance (3.2) is strongly motivated given how well the results we have obtained agree with data on jet $R_{\mathrm{AA}}$, the dijet asymmetry $A_{J}$ and, to this point most discriminatingly, the fragmentation function ratio. The hybrid approach has already provided us with a calculational framework within which we can test strongly coupled predictions for jet quenching by confronting them quantitatively with experimental measurements of jet observables. This demonstrates that this approach can now be used to explore and subsequently test new observables. Having the means to quantitatively confront new ideas, like for example the relationship between the centrality dependence of the ratio of the number of quarkinitiated jets and the number of gluon-initiated jets that remain in the final state, new observables, and new data is critical if we are eventually to understand the properties of the strongly coupled liquid quark-gluon plasma that Nature has served us. 


\section{Acknowledgments}

We are grateful to Paul Chesler, Peter Jacobs, Andreas Karch, Yen-Jie Lee, Al Mueller, Gunther Roland, Jesse Thaler, Xin-Nian Wang and Korinna Zapp for helpful conversations over the course of this work. KR is grateful to the CERN Theory Division for hospitality at the time this research was begun. The work of JCS was supported by a Ramón y Cajal fellowship. The work of JCS and DP was supported by the Marie Curie Career Integration Grant FP7-PEOPLE-2012-GIG-333786, by grants FPA2010-20807 and FPA2013-40360ERC from the Ministerio de Economía y Competitividad, Spain, by grant 2009SGR502 from the Generalitat de Catalunya and by the Consolider CPAN project. The work of DCG and KR was supported by the U.S. Department of Energy under cooperative research agreement DE-FG0205ER41360. The work of JGM was supported by Fundação para a Ciência e a Tecnologia (Portugal) under project CERN/FP/123596/2011 and contract 'Investigador FCT — Development Grant'.

Open Access. This article is distributed under the terms of the Creative Commons Attribution License (CC-BY 4.0), which permits any use, distribution and reproduction in any medium, provided the original author(s) and source are credited.

\section{References}

[1] ATLAS collaboration, Observation of a centrality-dependent dijet asymmetry in lead-lead collisions at $\sqrt{s_{\mathrm{NN}}}=2.77 \mathrm{TeV}$ with the ATLAS detector at the LHC, Phys. Rev. Lett. 105 (2010) 252303 [arXiv:1011.6182] [INSPIRE].

[2] CMS collaboration, Observation and studies of jet quenching in PbPb collisions at nucleon-nucleon center-of-mass energy $=2.76$ TeV, Phys. Rev. C 84 (2011) 024906 [arXiv:1102.1957] [INSPIRE].

[3] PHENIX collaboration, K. Adcox et al., Suppression of hadrons with large transverse momentum in central Au+Au collisions at $\sqrt{s_{\mathrm{NN}}}=130 \mathrm{GeV}$, Phys. Rev. Lett. 88 (2002) 022301 [nucl-ex/0109003] [INSPIRE].

[4] STAR collaboration, C. Adler et al., Centrality dependence of high $p_{T}$ hadron suppression in Au+Au collisions at $\sqrt{s}_{\mathrm{NN}}=130$ GeV, Phys. Rev. Lett. 89 (2002) 202301 [nucl-ex/0206011] [INSPIRE].

[5] U. Heinz and R. Snellings, Collective flow and viscosity in relativistic heavy-ion collisions, Ann. Rev. Nucl. Part. Sci. 63 (2013) 123 [arXiv:1301.2826] [InSPIRE].

[6] J. Casalderrey-Solana, H. Liu, D. Mateos, K. Rajagopal and U.A. Wiedemann, Gauge/string duality, hot QCD and heavy ion collisions, arXiv:1101.0618 [INSPIRE].

[7] R. Baier, Y.L. Dokshitzer, A.H. Mueller, S. Peigne and D. Schiff, Radiative energy loss of high-energy quarks and gluons in a finite volume quark-gluon plasma, Nucl. Phys. B 483 (1997) 291 [hep-ph/9607355] [INSPIRE].

[8] R. Baier, Y.L. Dokshitzer, A.H. Mueller and D. Schiff, Medium induced radiative energy loss: equivalence between the BDMPS and Zakharov formalisms, Nucl. Phys. B 531 (1998) 403 [hep-ph/9804212] [INSPIRE]. 
[9] M. Gyulassy, P. Levai and I. Vitev, Reaction operator approach to non-Abelian energy loss, Nucl. Phys. B 594 (2001) 371 [nucl-th/0006010] [INSPIRE].

[10] U.A. Wiedemann, Gluon radiation off hard quarks in a nuclear environment: opacity expansion, Nucl. Phys. B 588 (2000) 303 [hep-ph/0005129] [INSPIRE].

[11] X.-N. Wang and X.-F. Guo, Multiple parton scattering in nuclei: parton energy loss, Nucl. Phys. A 696 (2001) 788 [hep-ph/0102230] [INSPIRE].

[12] P.B. Arnold, G.D. Moore and L.G. Yaffe, Photon and gluon emission in relativistic plasmas, JHEP 06 (2002) 030 [hep-ph/0204343] [INSPIRE].

[13] P. Jacobs and X.-N. Wang, Matter in extremis: ultrarelativistic nuclear collisions at RHIC, Prog. Part. Nucl. Phys. 54 (2005) 443 [hep-ph/0405125] [inSPIRE].

[14] J. Casalderrey-Solana and C.A. Salgado, Introductory lectures on jet quenching in heavy ion collisions, Acta Phys. Polon. B 38 (2007) 3731 [arXiv:0712.3443] [inSPIRE].

[15] A. Majumder and M. Van Leeuwen, The theory and phenomenology of perturbative QCD based jet quenching, Prog. Part. Nucl. Phys. A 66 (2011) 41 [arXiv:1002.2206] [InSPIRE].

[16] Y. Mehtar-Tani, J.G. Milhano and K. Tywoniuk, Jet physics in heavy-ion collisions, Int. J. Mod. Phys. A 28 (2013) 1340013 [arXiv:1302.2579] [INSPIRE].

[17] S. Wicks, W. Horowitz, M. Djordjevic and M. Gyulassy, Elastic, inelastic and path length fluctuations in jet tomography, Nucl. Phys. A 784 (2007) 426 [nucl-th/0512076] [INSPIRE].

[18] C.P. Herzog, A. Karch, P. Kovtun, C. Kozcaz and L.G. Yaffe, Energy loss of a heavy quark moving through $N=4$ supersymmetric Yang-Mills plasma, JHEP 07 (2006) 013 [hep-th/0605158] [INSPIRE].

[19] J. Casalderrey-Solana and D. Teaney, Heavy quark diffusion in strongly coupled $N=4$ Yang-Mills, Phys. Rev. D 74 (2006) 085012 [hep-ph/0605199] [InSPIRE].

[20] S.S. Gubser, Drag force in AdS/CFT, Phys. Rev. D 74 (2006) 126005 [hep-th/0605182] [INSPIRE].

[21] H. Liu, K. Rajagopal and U.A. Wiedemann, An AdS/CFT calculation of screening in a hot wind, Phys. Rev. Lett. 98 (2007) 182301 [hep-ph/0607062] [INSPIRE].

[22] P.M. Chesler, K. Jensen, A. Karch and L.G. Yaffe, Light quark energy loss in strongly-coupled $N=4$ supersymmetric Yang-Mills plasma, Phys. Rev. D 79 (2009) 125015 [arXiv:0810.1985] [INSPIRE].

[23] S.S. Gubser, D.R. Gulotta, S.S. Pufu and F.D. Rocha, Gluon energy loss in the gauge-string duality, JHEP 10 (2008) 052 [arXiv: 0803.1470] [INSPIRE].

[24] P. Arnold and D. Vaman, Jet quenching in hot strongly coupled gauge theories revisited: 3-point correlators with gauge-gravity duality, JHEP 10 (2010) 099 [arXiv: 1008.4023] [INSPIRE].

[25] P. Arnold and D. Vaman, Jet quenching in hot strongly coupled gauge theories simplified, JHEP 04 (2011) 027 [arXiv: 1101.2689] [INSPIRE].

[26] M. Chernicoff, J.A. Garcia, A. Guijosa and J.F. Pedraza, Holographic lessons for quark dynamics, J. Phys. G 39 (2012) 054002 [arXiv:1111.0872] [INSPIRE].

[27] P.M. Chesler, Y.-Y. Ho and K. Rajagopal, Shining a gluon beam through quark-gluon plasma, Phys. Rev. D 85 (2012) 126006 [arXiv:1111.1691] [INSPIRE]. 
[28] C. Marquet and T. Renk, Jet quenching in the strongly-interacting quark-gluon plasma, Phys. Lett. B 685 (2010) 270 [arXiv:0908.0880] [INSPIRE].

[29] B. Betz, M. Gyulassy and G. Torrieri, Sensitivity of azimuthal jet tomography to early time energy-loss at RHIC and LHC, J. Phys. G 38 (2011) 124153 [arXiv:1106.4564] [INSPIRE].

[30] A. Ficnar, J. Noronha and M. Gyulassy, Falling strings and light quark jet quenching at LHC, Nucl. Phys. A 910-911 (2013) 252 [arXiv:1208.0305] [InSPIRE].

[31] B. Betz and M. Gyulassy, Constraints on the path-length dependence of jet quenching in nuclear collisions at RHIC and LHC, JHEP 08 (2014) 090 [Erratum ibid. 10 (2014) 043] [arXiv: 1404.6378] [INSPIRE].

[32] J. Casalderrey-Solana, J.G. Milhano and P.Q. Arias, Out of medium fragmentation from long-lived jet showers, Phys. Lett. B 710 (2012) 175 [arXiv:1111.0310] [INSPIRE].

[33] J. Casalderrey-Solana, J.G. Milhano and U.A. Wiedemann, Jet quenching via jet collimation, J. Phys. G 38 (2011) 035006 [arXiv: 1012.0745] [INSPIRE].

[34] J.-P. Blaizot, E. Iancu and Y. Mehtar-Tani, Medium-induced QCD cascade: democratic branching and wave turbulence, Phys. Rev. Lett. 111 (2013) 052001 [arXiv:1301.6102] [INSPIRE].

[35] P.M. Chesler, M. Lekaveckas and K. Rajagopal, Heavy quark energy loss far from equilibrium in a strongly coupled collision, JHEP 10 (2013) 013 [arXiv:1306.0564] [INSPIRE].

[36] J. Casalderrey-Solana, M.P. Heller, D. Mateos and W. van der Schee, From full stopping to transparency in a holographic model of heavy ion collisions, Phys. Rev. Lett. 111 (2013) 181601 [arXiv: 1305.4919] [INSPIRE].

[37] Y. Hatta, E. Iancu and A.H. Mueller, Jet evolution in the $N=4$ SYM plasma at strong coupling, JHEP 05 (2008) 037 [arXiv:0803.2481] [INSPIRE].

[38] P.M. Chesler, K. Jensen and A. Karch, Jets in strongly-coupled $N=4$ super Yang-Mills theory, Phys. Rev. D 79 (2009) 025021 [arXiv:0804.3110] [InSPIRE].

[39] P. Arnold and D. Vaman, Some new results for 'jet' stopping in AdS/CFT: long version, J. Phys. G 38 (2011) 124175 [arXiv:1106.1680] [InSPIRE].

[40] A. Ficnar, AdS/CFT energy loss in time-dependent string configurations, Phys. Rev. D 86 (2012) 046010 [arXiv:1201.1780] [INSPIRE].

[41] P. Arnold, P. Szepietowski and D. Vaman, Coupling dependence of jet quenching in hot strongly-coupled gauge theories, JHEP 07 (2012) 024 [arXiv: 1203.6658] [INSPIRE].

[42] P. Arnold, P. Szepietowski, D. Vaman and G. Wong, Tidal stretching of gravitons into classical strings: application to jet quenching with AdS/CFT, JHEP 02 (2013) 130 [arXiv: 1212.3321] [INSPIRE].

[43] A. Ficnar and S.S. Gubser, Finite momentum at string endpoints, Phys. Rev. D 89 (2014) 026002 [arXiv: 1306.6648] [INSPIRE].

[44] A. Ficnar, S.S. Gubser and M. Gyulassy, Shooting string holography of jet quenching at RHIC and LHC, Phys. Lett. B 738 (2014) 464 [arXiv:1311.6160] [InSPIRE].

[45] P.M. Chesler and K. Rajagopal, Jet quenching in strongly coupled plasma, Phys. Rev. D 90 (2014) 025033 [arXiv:1402.6756] [INSPIRE]. 
[46] P.K. Kovtun and A.O. Starinets, Quasinormal modes and holography, Phys. Rev. D 72 (2005) 086009 [hep-th/0506184] [INSPIRE].

[47] J. Casalderrey-Solana, E.V. Shuryak and D. Teaney, Conical flow induced by quenched QCD jets, J. Phys. Conf. Ser. 27 (2005) 22 [Nucl. Phys. A 774 (2006) 577] [hep-ph/0411315] [INSPIRE].

[48] W.A. Horowitz and M. Gyulassy, Testing AdS/CFT drag and $p Q C D$ heavy quark energy loss, J. Phys. G 35 (2008) 104152 [arXiv:0804.4330] [InSPIRE].

[49] W.A. Horowitz and M. Gyulassy, The surprising transparency of the sQGP at LHC, Nucl. Phys. A 872 (2011) 265 [arXiv:1104.4958] [INSPIRE].

[50] W.A. Horowitz and M. Gyulassy, Quenching and tomography from RHIC to LHC, J. Phys. G 38 (2011) 124114 [arXiv:1107.2136] [INSPIRE].

[51] F. Dominguez, C. Marquet, A.H. Mueller, B. Wu and B.-W. Xiao, Comparing energy loss and p-perpendicular - broadening in perturbative QCD with strong coupling $N=4 S Y M$ theory, Nucl. Phys. A 811 (2008) 197 [arXiv:0803.3234] [INSPIRE].

[52] JET collaboration, K.M. Burke et al., Extracting the jet transport coefficient from jet quenching in high-energy heavy-ion collisions, Phys. Rev. C 90 (2014) 014909 [arXiv: 1312.5003] [INSPIRE].

[53] J. Casalderrey-Solana and X.-N. Wang, Energy dependence of jet transport parameter and parton saturation in quark-gluon plasma, Phys. Rev. C 77 (2008) 024902 [arXiv: 0705.1352] [INSPIRE].

[54] E. Iancu, The non-linear evolution of jet quenching, JHEP 10 (2014) 095 [arXiv:1403.1996] [INSPIRE].

[55] J.-P. Blaizot and Y. Mehtar-Tani, Renormalization of the jet-quenching parameter, Nucl. Phys. A 929 (2014) 202 [arXiv:1403.2323] [inSPIRE].

[56] T. Renk, Parton shower evolution in a 3D hydrodynamical medium, Phys. Rev. C 78 (2008) 034908 [arXiv:0806.0305] [inSPIRE].

[57] I.P. Lokhtin, L.V. Malinina, S.V. Petrushanko, A.M. Snigirev, I. Arsene and K. Tywoniuk, Heavy ion event generator HYDJET++ (HYDrodynamics plus JETs), Comput. Phys. Commun. 180 (2009) 779 [arXiv:0809.2708] [INSPIRE].

[58] B. Schenke, C. Gale and S. Jeon, MARTINI: an event generator for relativistic heavy-ion collisions, Phys. Rev. C 80 (2009) 054913 [arXiv: 0909. 2037] [INSPIRE].

[59] N. Armesto, L. Cunqueiro and C.A. Salgado, Q-PYTHIA: a medium-modified implementation of final state radiation, Eur. Phys. J. C 63 (2009) 679 [arXiv:0907.1014] [INSPIRE].

[60] I.P. Lokhtin, A.V. Belyaev and A.M. Snigirev, Jet quenching pattern at LHC in PYQUEN model, Eur. Phys. J. C 71 (2011) 1650 [arXiv:1103.1853] [INSPIRE].

[61] K.C. Zapp, JEWEL 2.0.0: directions for use, Eur. Phys. J. C 74 (2014) 2762 [arXiv: 1311.0048] [INSPIRE].

[62] T. Sjöstrand, S. Mrenna and P.Z. Skands, A brief introduction to PYTHIA 8.1, Comput. Phys. Commun. 178 (2008) 852 [arXiv:0710.3820] [INSPIRE].

[63] K.J. Eskola, H. Paukkunen and C.A. Salgado, EPS09: a new generation of NLO and LO nuclear parton distribution functions, JHEP 04 (2009) 065 [arXiv:0902.4154] [INSPIRE]. 
[64] CMS collaboration, Studies of dijet transverse momentum balance and pseudorapidity distributions in $p P b$ collisions at $\sqrt{s_{\mathrm{NN}}}=5.02 \mathrm{TeV}$, Eur. Phys. J. C 74 (2014) 2951 [arXiv:1401.4433] [INSPIRE].

[65] R.J. Fries, B. Müller, C. Nonaka and S.A. Bass, Hadronization in heavy ion collisions: recombination and fragmentation of partons, Phys. Rev. Lett. 90 (2003) 202303 [nucl-th/0301087] [INSPIRE].

[66] D. Molnar and S.A. Voloshin, Elliptic flow at large transverse momenta from quark coalescence, Phys. Rev. Lett. 91 (2003) 092301 [nucl-th/0302014] [INSPIRE].

[67] Y. Mehtar-Tani, C.A. Salgado and K. Tywoniuk, Anti-angular ordering of gluon radiation in QCD media, Phys. Rev. Lett. 106 (2011) 122002 [arXiv:1009.2965] [INSPIRE].

[68] Y. Mehtar-Tani, C.A. Salgado and K. Tywoniuk, Jets in QCD media: from color coherence to decoherence, Phys. Lett. B 707 (2012) 156 [arXiv:1102.4317] [INSPIRE].

[69] J. Casalderrey-Solana and E. Iancu, Interference effects in medium-induced gluon radiation, JHEP 08 (2011) 015 [arXiv:1105.1760] [INSPIRE].

[70] A. Beraudo, J.G. Milhano and U.A. Wiedemann, Medium-induced color flow softens hadronization, Phys. Rev. C 85 (2012) 031901 [arXiv:1109.5025] [INSPIRE].

[71] A. Beraudo, J.G. Milhano and U.A. Wiedemann, The contribution of medium-modified color flow to jet quenching, JHEP 07 (2012) 144 [arXiv: 1204.4342] [INSPIRE].

[72] P. Aurenche and B.G. Zakharov, Jet color chemistry and anomalous baryon production in AA-collisions, Eur. Phys. J. C 71 (2011) 1829 [arXiv:1109.6819] [INSPIRE].

[73] G. Soyez, A simple description of jet cross-section ratios, Phys. Lett. B 698 (2011) 59 [arXiv:1101.2665] [INSPIRE].

[74] T. Hirano, P. Huovinen and Y. Nara, Elliptic flow in $P b+P b$ collisions at $\sqrt{s_{\mathrm{NN}}}=2.76$ TeV: hybrid model assessment of the first data, Phys. Rev. C 84 (2011) 011901 [arXiv:1012.3955] [INSPIRE].

[75] A. Bazavov et al., Equation of state and QCD transition at finite temperature, Phys. Rev. D 80 (2009) 014504 [arXiv:0903.4379] [INSPIRE].

[76] A. Bialas, M. Bleszynski and W. Czyz, Multiplicity distributions in nucleus-nucleus collisions at high-energies, Nucl. Phys. B 111 (1976) 461 [InSPIRE].

[77] M.L. Miller, K. Reygers, S.J. Sanders and P. Steinberg, Glauber modeling in high energy nuclear collisions, Ann. Rev. Nucl. Part. Sci. 57 (2007) 205 [nucl-ex/0701025] [InSPIRE].

[78] M. Cacciari, G.P. Salam and G. Soyez, FastJet user manual, Eur. Phys. J. C 72 (2012) 1896 [arXiv:1111.6097] [INSPIRE].

[79] M. Cacciari, G.P. Salam and G. Soyez, The anti- $k_{t}$ jet clustering algorithm, JHEP 04 (2008) 063 [arXiv:0802.1189] [INSPIRE].

[80] CMS collaboration, Nuclear modification factor of high transverse momentum jets in $\mathrm{PbPb}$ collisions at sqrts $_{\mathrm{NN}}=2.76 \mathrm{Te}$, CMS-PAS-HIN-12-004, CERN, Geneva Switzerland (2012).

[81] ATLAS collaboration, Measurement of the jet radius and transverse momentum dependence of inclusive jet suppression in lead-lead collisions at $\sqrt{s_{\mathrm{NN}}}=2.76$ TeV with the ATLAS detector, Phys. Lett. B 719 (2013) 220 [arXiv:1208.1967] [INSPIRE]. 
[82] ALICE collaboration, Measurement of charged jet suppression in $\mathrm{Pb}-\mathrm{Pb}$ collisions at $\sqrt{s_{\mathrm{NN}}}=2.76 \mathrm{TeV}$, JHEP 03 (2014) 013 [arXiv:1311.0633] [INSPIRE].

[83] STAR collaboration, J. Rusnak, Inclusive spectrum of charged jets in central Au+Au collisions at $\sqrt{s_{\mathrm{NN}}}=200 \mathrm{GeV}$ by STAR collaboration, talk at the conference Hard Probes 2013, Cape Town South Africa November 2013 [PoS (EPS-HEP 2013)191] [INSPIRE].

[84] CMS collaboration, Jet momentum dependence of jet quenching in $\mathrm{PbPb}$ collisions at $\sqrt{s_{\mathrm{NN}}}=2.76 \mathrm{TeV}$, Phys. Lett. B 712 (2012) 176 [arXiv:1202.5022] [INSPIRE].

[85] Y. Yilmaz, Jet quenching in heavy-ion collisions at LHC with CMS detector, Ph.D. thesis, http://web.mit.edu/mithig/theses/Yetkin-Yilmaz-thesis.pdf, Massachusetts Institute of Technology, U.S.A. (2013) [CMS-TS-2013-035] [INSPIRE].

[86] T. Renk, On the sensitivity of the dijet asymmetry to the physics of jet quenching, Phys. Rev. C 85 (2012) 064908 [arXiv: 1202.4579] [INSPIRE].

[87] CMS collaboration, Measurement of jet fragmentation into charged particles in pp and $\mathrm{PbPb}$ collisions at $\sqrt{s_{\mathrm{NN}}}=2.76 \mathrm{TeV}$, JHEP 10 (2012) 087 [arXiv:1205.5872] [INSPIRE].

[88] CMS collaboration,, Measurement of jet fragmentation in $\mathrm{PbPb}$ and pp collisions at $\sqrt{s_{\mathrm{NN}}}=2.76 \mathrm{TeV}$, CMS-HIN-12-013, CERN, Geneva Switzerland (2012) [Phys. Rev. C 90 (2014) 024908] [arXiv:1406.0932] [INSPIRE].

[89] ATLAS collaboration, Measurement of inclusive jet charged particle fragmentation functions in $\mathrm{Pb}+\mathrm{Pb}$ collisions at $\sqrt{s_{\mathrm{NN}}}=2.76 \mathrm{TeV}$ with the ATLAS detector, ATLAS-CONF-2012-115, CERN, Geneva Switzerland (2012).

[90] CMS collaboration, Studies of jet quenching using isolated-photon+jet correlations in $\mathrm{PbPb}$ and pp collisions at $\sqrt{s_{\mathrm{NN}}}=2.76 \mathrm{TeV}$, Phys. Lett. B 718 (2013) 773 [arXiv:1205.0206] [INSPIRE].

[91] F. D'Eramo, M. Lekaveckas, H. Liu and K. Rajagopal, Momentum broadening in weakly coupled quark-gluon plasma (with a view to finding the quasiparticles within liquid quark-gluon plasma), JHEP 05 (2013) 031 [arXiv:1211.1922] [INSPIRE].

[92] H. Liu, K. Rajagopal and U.A. Wiedemann, Calculating the jet quenching parameter from AdS/CFT, Phys. Rev. Lett. 97 (2006) 182301 [hep-ph/0605178] [INSPIRE].

[93] F. D'Eramo, H. Liu and K. Rajagopal, Transverse momentum broadening and the jet quenching parameter, redux, Phys. Rev. D 84 (2011) 065015 [arXiv:1006.1367] [InSPIRE].

[94] J. Casalderrey-Solana, Y. Mehtar-Tani, C.A. Salgado and K. Tywoniuk, New picture of jet quenching dictated by color coherence, Phys. Lett. B 725 (2013) 357 [arXiv:1210.7765] [INSPIRE].

[95] A. Banfi, G.P. Salam and G. Zanderighi, Infrared safe definition of jet flavor, Eur. Phys. J. C 47 (2006) 113 [hep-ph/0601139] [INSPIRE].

[96] J. Gallicchio and M.D. Schwartz, Quark and gluon tagging at the LHC, Phys. Rev. Lett. 107 (2011) 172001 [arXiv:1106.3076] [INSPIRE].

[97] CMS collaboration, Search for a Higgs boson in the decay channel $H \rightarrow Z Z^{*} \rightarrow q \bar{q} \ell^{-} \ell^{+}$in pp collisions at $\sqrt{s}=7 \mathrm{TeV}$, JHEP 04 (2012) 036 [arXiv:1202.1416] [INSPIRE].

[98] D. Krohn, M.D. Schwartz, T. Lin and W.J. Waalewijn, Jet charge at the LHC, Phys. Rev. Lett. 110 (2013) 212001 [arXiv: 1209.2421] [INSPIRE]. 
[99] J. Gallicchio and M.D. Schwartz, Quark and gluon jet substructure, JHEP 04 (2013) 090 [arXiv:1211.7038] [INSPIRE].

[100] A.J. Larkoski, G.P. Salam and J. Thaler, Energy correlation functions for jet substructure, JHEP 06 (2013) 108 [arXiv:1305.0007] [INSPIRE].

[101] CMS collaboration, Evidence of b-jet quenching in PbPb collisions at $\sqrt{s_{\mathrm{NN}}}=2.76 \mathrm{TeV}$, Phys. Rev. Lett. 113 (2014) 132301 [Erratum ibid. 115 (2015) 029903] [arXiv:1312.4198] [INSPIRE].

[102] H. Liu, K. Rajagopal and U.A. Wiedemann, Wilson loops in heavy ion collisions and their calculation in AdS/CFT, JHEP 03 (2007) 066 [hep-ph/0612168] [INSPIRE].

[103] S.S. Gubser, Momentum fluctuations of heavy quarks in the gauge-string duality, Nucl. Phys. B 790 (2008) 175 [hep-th/0612143] [INSPIRE].

[104] J. Casalderrey-Solana and D. Teaney, Transverse momentum broadening of a fast quark in a $N=4$ Yang-Mills plasma, JHEP 04 (2007) 039 [hep-th/0701123] [INSPIRE]. 